\title{
C-Glycoside D-galacturonates suitable as glycosyl acceptors for the synthesis of allyl $C$-homo- and rhamno-galacturonan modules ${ }^{1}$
}

\author{
Mahmoud Farouk, ${ }^{a}{ }$ Dirk Michalik, ${ }^{b}$ Alexander Villinger, ${ }^{b}$ and Christian Vogel ${ }^{b}$ \\ ${ }^{a}$ College of Sciences and Humanities, Salman Bin Abdalaziz University, P. O. Box 83, \\ Alkharj 11942, Saudi Arabia \\ ${ }^{b}$ University of Rostock, Institute of Chemistry, Department of Organic Chemistry, \\ Albert-Einstein-Strasse 3a, D-18059 Rostock, Germany \\ E-mail:ㅍa23@yahoo.com
}

Dedicated to Prof. Richard R. Schmidt on the occasion of his 78th anniversary

\begin{abstract}
Methyl 2,3-di-O-benzyl-1-deoxy-1-(prop-2-enyl)- $\alpha$-D-galactopyranuronate and benzyl 2,3-di- $O$ benzyl-1-deoxy-1-(prop-2-enyl)- $\alpha$-D-galactopyranuronate were prepared as glycosyl acceptors. The glycosyl donors benzyl 4- $O$-acetyl-2,3-di- $O$-benzyl-1-(trichloroacetimidoyloxy)- $\alpha$-Dgalactopyranosyluronate and benzyl $4-O$-acetyl-2,3-di- $O$-benzyl-1-(trichloroacetimidoyloxy)- $\beta$ D-galactopyranosyluronate were synthesized in $98 \%$ and $76 \%$ yields respectively. Disaccharides methyl (methyl 4-O-acetyl-2,3-di-O-benzyl- $\alpha / \beta$-D-galactopyranosyluronate)-( $1 \rightarrow 4)-2,3$-di-Obenzyl-1-deoxy-1-(prop-2-enyl)- $\alpha$-D-galactopyranuronate and benzyl (benzyl 4-O-acetyl-2,3-diO-benzyl- $\alpha / \beta$-D-galactopyranosyluronate)-( $1 \rightarrow 4)-2,3$-di-O-benzyl-1-deoxy-1-(prop-2-enyl)- $\alpha$-Dgalactopyranuronate were synthesized in $53 \%$ and $44 \%$ yields respectively and with moderate stereoselectivity. The synthesis of 1,2- $O$-acetyl-3,4-di- $O$-benzyl-L-rhamnopyranose $(\alpha / \beta)$ and 2$O$-acetyl-1-bromo-3,4-di- $O$-benzyl- $\alpha$-L-rhamnopyranosyl suitable as glycosyl donors is described. The disaccharide methyl (2-O-acetyl-3,4-di- $O$-benzyl- $\alpha$-L-rhamnopyranosyl)-( $1 \rightarrow 4)-$ 2,3-di- $O$-benzyl-1-deoxy-1-(prop-2-enyl)- $\alpha$-D-galactopyranuronate, and benzyl (2-O-acetyl-3,4di- $O$-benzyl- $\alpha$-L-rhamnopyranosyl)-( $1 \rightarrow 4$ )-2,3-di- $O$-benzyl-1-deoxy-1-(prop-2-enyl)- $\alpha$-D-galactopyranuronate were obtained in $54 \%$ and $56 \%$ yield respectively.
\end{abstract}

Keywords: C-glycoside, D-galacturonic acid, allyl $C$-homogalacturonan, allyl $C$-rhamnogalacturonan 


\section{Introduction}

In order to develop a program directed at the synthesis of homo- and rhamnogalacturonan disaccharide mimics, we looked for an option to use $\alpha$ - $C$-galacturonate acceptors ${ }^{1,2}$ in glycosidic reactions to form higher oligomers of defined structure in our ongoing program for the synthesis of pectin fragments by a modular design principle. ${ }^{1-3}$

\section{Results and Discussion}

In our previous paper ${ }^{2}$ methyl (4) and benzyl (5) glycosyl acceptors were obtained by different routes starting from D-galactose and D-galacturonic acid respectively. Exploring several protecting group manipulations, an effective route was found for the preparation of the key intermediate $\mathbf{3}$ (Scheme 1) through the isopropylidene compound $\mathbf{1}$ and its benzylation under basic conditions, where no $\beta$-elimination was observed. ${ }^{2,3}$ Selective benzylation via 3,4-Obenzylstannyl intermediates resulted in methyl (4) and benzyl (5) glycosyl acceptors, both suitable as acceptors in glycosylation reactions.

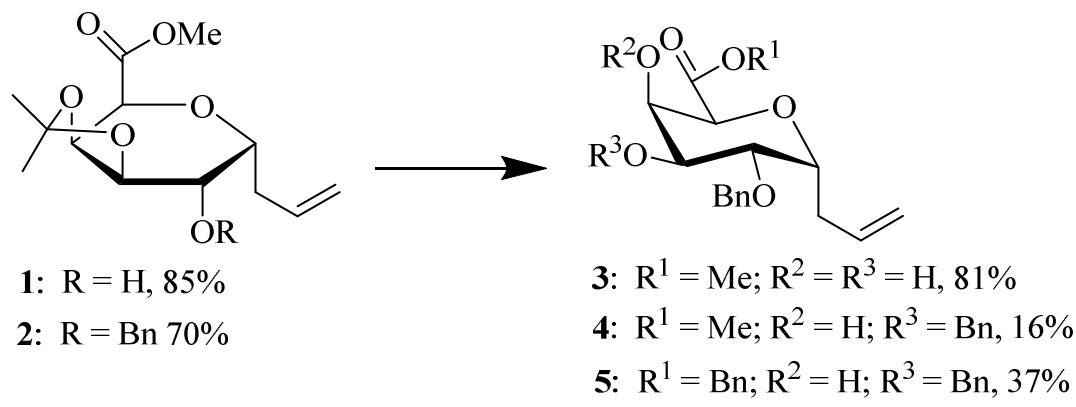

\section{Scheme 1}

For the preparation of the galacturonate trichloroacetimidate donors, the introduction of the trichloroacetimidate group at the anomeric centre of $\mathbf{1 1}^{4,5}$ was re-examined (Scheme 2). In our investigations ${ }^{1}$, the introduction of the trichloroacetimidate group at the anomeric center of $\mathbf{9}$ in the presence of DBU gave a 3:1 mixture of the $\alpha$ - and $\beta$-trichloroacetimidates $(\mathbf{1 1} \alpha, \beta)$ in $98 \%$ total yield. After HPLC column chromatography the $\alpha$-trichloroacetimidate $\left(\mathrm{R}_{f} .0 .39,73 \%\right)$ and the $\beta$-trichloroacetimidate $\left(\mathrm{R}_{f} \quad 0.27,25 \%\right)$ were characterized by NMR spectroscopy. For H-1, the smaller coupling constant $\left(J_{1,2}=3.5 \mathrm{~Hz}\right)$ for compound $13 \alpha$ and the larger $\left(J_{1,2}=8.2 \mathrm{~Hz}\right)$ for the trichloroacetimidate $\mathbf{1 3} \beta$ match the observed results. 


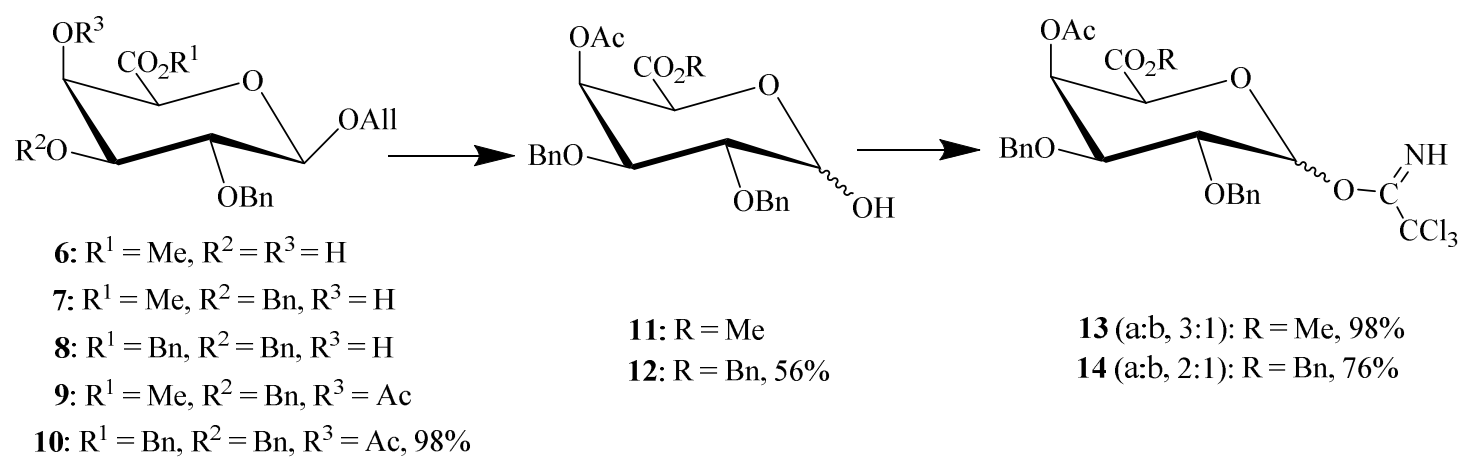

\section{Scheme 2}

In general, benzyl esters are less stable than the corresponding methyl esters. On the other hand, benzyl esters can be removed easily by hydrogenation. In order to get a defined pattern of protected and unprotected carboxylic esters in pectin fragments, both methyl and benzyl esters were prepared. ${ }^{6,7}$ Thus, it was of interest to incorporate galacturonate $\mathbf{8}$, protected as the benzyl ester, into our program for the synthesis of the glycosyl donors $14 \boldsymbol{\alpha} / \boldsymbol{\beta}$. Therefore, the $4-O-$ position was acetylated with acetic anhydride in dry pyridine to provide $\mathbf{1 0}$ in $98 \%$ yield. Deallylation with the aid of palladium(II) chloride $^{8}$ produced, surprisingly, only the $\alpha$-product $\mathbf{1 2}$ in $56 \%$ yield (Scheme 2).

Regioselective benzylation of $\mathbf{6}$ via 3,4-O-butylstannyl intermediates gave galacturonates 7 and 8 in $80 \%$ overall yield (ratio 3:1). The galacturonate 7 was used as a glycosyl acceptor for the synthesis of homo- and rhamnogalacturonan oligosaccharides. ${ }^{3,9}$

In the ${ }^{1} \mathrm{H}$ NMR (500 MHz) spectrum, the acetylation of 6 caused the expected downfield shift to the H-4 ring proton signal from $\delta 4.32 \mathrm{ppm}(\mathbf{8})$ to $5.82 \mathrm{ppm}(\mathbf{1 0})$. In the ${ }^{13} \mathrm{C} \mathrm{NMR}$ spectrum, the signal at $\delta 20.53 \mathrm{ppm}$ and $169.68 \mathrm{ppm}$ confirmed the presence of the acetyl group at the 4-position. The deallylation was evidenced by the expected upfield shift of the H-1 ring proton signal from $\delta 4.48 \mathrm{ppm}(\mathbf{1 0})$ to $5.40 \mathrm{ppm}(\mathbf{1 2})$. The small coupling constant $J_{1,2}=3.5 \mathrm{~Hz}$ of $\mathrm{H}-1$ and the $\mathrm{C}-1$ signal at $\delta 92.18 \mathrm{ppm}$ are caused by the $\alpha$-configuration of $\mathbf{8}$.

The introduction of the trichloroacetimidate group at the anomeric center of $\mathbf{1 1}$ in the presence of DBU produced the $\alpha$-D-galactopyranosyl trichloroacetimidate $13 \alpha\left(\mathrm{R}_{f} 0.76\right)$ and the $\beta$-trichloroacetimidate $12 \beta\left(\mathrm{R}_{f} 0.71\right)$. After HPLC chromatography compounds $13 \alpha$ and $13 \beta$ were obtained in pure in $76 \%$ total yield (ratio 2:1). In the ${ }^{1} \mathrm{H}$ NMR $(500 \mathrm{MHz}$ ) spectrum the small coupling constant $J_{1,2}=3.5 \mathrm{~Hz}$ for compound $\mathbf{1 3} \alpha$, and the considerably larger coupling constant $J_{1,2}=8.2 \mathrm{~Hz}$ for compound $13 \beta$ confirmed the proposed structure. Moreover, ${ }^{13} \mathrm{C}$ NMR signals at $\delta 94.54 \mathrm{ppm}$ for compound $\mathbf{1 3} \alpha$, and at $\delta 97.78 \mathrm{ppm}$ for compound $\mathbf{1 3} \beta$ assured the stereochemistry at the anomeric center of the glycosyl donor $13 \alpha$ and $13 \beta$ respectively.

The coupling of galacturonate acceptor 4 with a slight excess of the $\alpha$-configured donor $13 \alpha$ was promoted by trimethylsilyl trifluoromethanesulfonate (TMSOTf). ${ }^{3,11,12}$ Standard work-up of 
the reaction mixture provided the $(1 \rightarrow 4)$-linked disaccharides $16 \alpha$ and $16 \beta$ in $53 \%$ total yield in a ratio 3:1 (Scheme 3). The same result was observed when 13 $\beta$ was used as glycosyl donor.

Although the $\mathrm{R}_{f}$. values of the resulting diaccharides were so close together, the disaccharides $15 \alpha$ and $15 \beta$ were successfully separated by using gradient HPLC. Still, both of the separated fractions $15 \alpha$ and $15 \beta$ contained traces of the corresponding anomer. The ${ }^{1} \mathrm{H}$ NMR spectra of $15 \alpha$ showed a doublet signal at $\delta 5.16 \mathrm{ppm}$ with vicinal coupling constant $J_{1^{\prime}, 2^{\prime}}=3.5 \mathrm{~Hz}$ for $\mathrm{H}-1^{\prime}$, and a triple doublet signal at $\delta 4.24$ with vicinal coupling constants $J_{1,2}=2.5 \mathrm{~Hz}, J_{1, \mathrm{Ha}}=6.0 \mathrm{~Hz}$, $J_{1, \mathrm{Hb}}=8.5 \mathrm{~Hz}$ for $\mathrm{H}-1$. In addition, the ${ }^{13} \mathrm{C} \mathrm{NMR}$ signal for $\mathrm{C}-1^{\prime}$ was found to fall within the expected range of $\delta 98.80 \mathrm{~Hz}$ with $J_{\mathrm{C}-1, \mathrm{H}-1^{\prime}}=171.0 \mathrm{~Hz}$ coupling constant for the $\alpha$-coupled disaccharide $15 \alpha$. In the case of $15 \beta$, the value of the $\mathrm{C}-1$ ' signal, which was determined at $\delta 102 \mathrm{~Hz}$, matches the $\beta$-linked disaccharide. Subsequent experiments have shown that the $\alpha$-or $\beta$-configured trichloroacetimidate group at the anomeric center of $13 \alpha / \beta$ exerted no influence on the outcome of stereoselectivity of the glycosylations investigated here. ${ }^{3,14}$ For that reason, in the subsequent experiments we used the $\alpha / \beta$ mixture of $13 \alpha / \beta$.
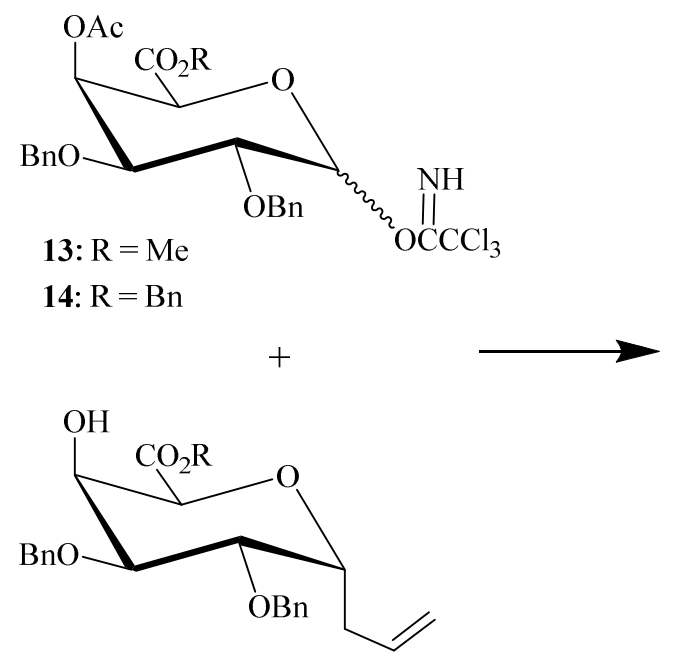

4: $\mathrm{R}=\mathrm{Me}$

5: $\mathrm{R}=\mathrm{Bn}$

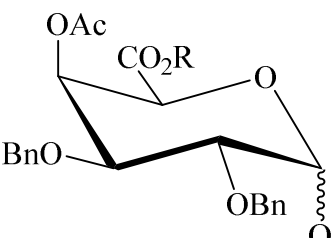

15: $\mathrm{R}=\mathrm{Me}$

16: $\mathrm{R}=\mathrm{Bn}$

\section{Scheme 3}

The coupling of glycosyl acceptor 5 with a slight excess of the $14 \alpha / \beta$ was initiated by TMSOTf as a glycosylation promoter. ${ }^{3,11,12}$ Standard work-up of the reaction mixture provided the $(1 \rightarrow 4)$-linked disaccharides $16 \alpha$ and $\mathbf{1 6} \beta$ in $44 \%$ total yield in a ratio of 2:1 (Scheme 3). Unfortunately, the $\alpha$ - and $\beta$-coupled disaccharides $16 \alpha$ and $16 \beta$ were difficult to separate this time. The ${ }^{13} \mathrm{C}$ NMR showed the signal for $\mathrm{C}-1$ ' has the expected range of $\delta 99.0 \mathrm{ppm}$ for the $\alpha$ coupled disaccharide $16 \alpha$, whereas the $\beta$-coupled disaccharide $16 \beta$ showed a signal at $\delta 102.3$ ppm. The other ${ }^{1} \mathrm{H}$ and ${ }^{13} \mathrm{C}$ NMR data were also fully consistent with the assigned structures.

In connection with the synthesis of rhamnogalacturonan fragments type I, we required a simple approach to benzylated rhamnopyranosides with an $O$-acetyl group at 2-postion. To 
achieve this, acetobromorhamnose 17 was prepared according to the literature. ${ }^{14}$ Methyl orthoester 18 was prepared in $81 \%$ yield by the reaction of bromide 17 with dry EtOH in the presence of 2,4,6-collidine and tetrabutylammonium bromide (Scheme 4). ${ }^{15,16}$ The structure of the obtained orthoester 18 was supported by the analytical data. ${ }^{17}$ Compound $\mathbf{1 8}$ was then deacetylated by the Zemplén procedure to give 18, but the subsequent neutralization decreased the yield of $\mathbf{2 0}$ dramatically. Therefore, deacetylation of orthoester $\mathbf{1 8}$ and the benzylation of the resulting compound $\mathbf{2 1}$ under basic conditions were carried out without further purification of $\mathbf{2 0}$. Instead of using Zemplèn conditions, deacetylation was achieved by refluxing $\mathbf{1 8}$ with $\mathrm{KOH}$ in dry toluene. ${ }^{18}$

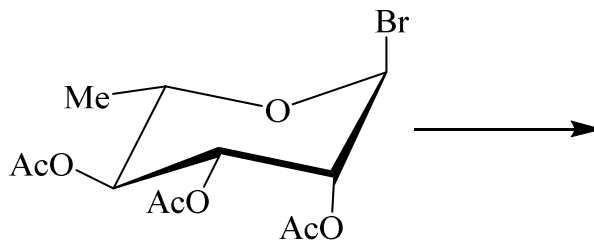

17

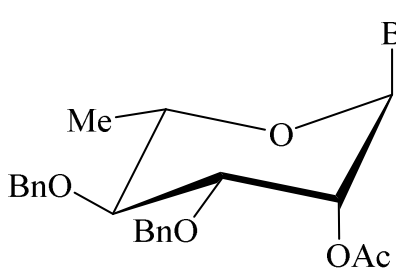

22

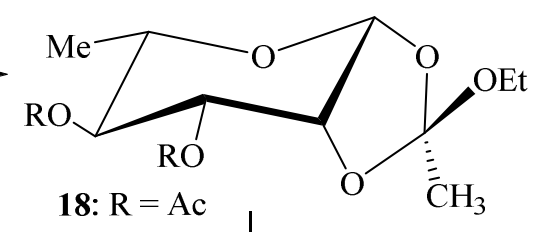

19: $\mathrm{R}=\mathrm{H}$

20: $\mathrm{R}=\mathrm{Bn}$
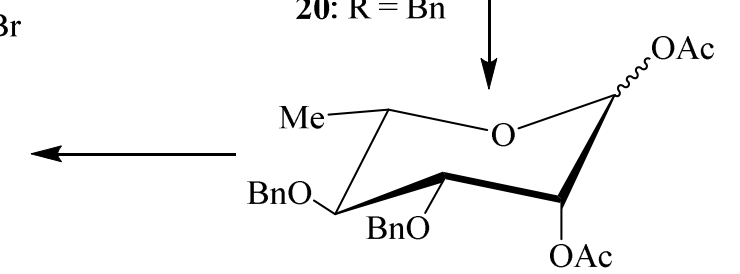

21

\section{Scheme 4}

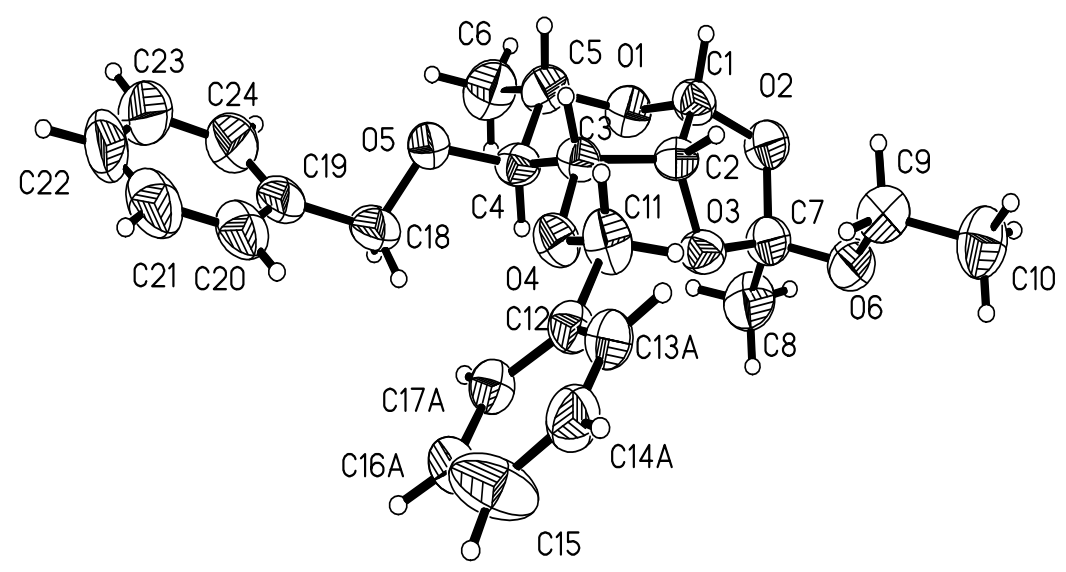

Figure 1. An ORTEP diagram of compound 20, the aromatic ring of the benzyl group at $\mathrm{O} 4$ is disordered over two positions around the C11-C12 axis. Only one set of atoms is shown in Figure 1, the other is omitted for clarity. 
After addition of benzyl chloride and classical work up orthoester $\mathbf{2 0}$ was obtained in $82 \%$ yield as colorless crystals suitable for X-ray investigation (Figure 1). NOESY studies (Figure 2) were employed to address the conformation and the absolute configuration of the orthoester 20. In the ${ }^{1} \mathrm{H}-\mathrm{NMR}$ spectrum of $\mathbf{2 0}$ the exchange of the acetyl groups at $O-3$ and $O-4$ positions by benzyl groups caused a significant upfield shift ( $c a .1 .5 \mathrm{ppm})$ of the geminal ring proton, with respect to the precursor $17 .{ }^{17}$ Furthermore, the $\mathrm{H}-1$ signal of $\mathbf{2 0}$ appears at relatively high field $\delta$ $5.26 \mathrm{ppm}$ with a coupling constant $J_{1,2}=2.5 \mathrm{~Hz}$. The NOESY spectrum (Figure 2) shows correlation between $\mathrm{H} 1 \leftrightarrow \mathrm{H} 2, \mathrm{H} 1 \leftrightarrow \mathrm{H} 3, \quad \mathrm{H} 1 \leftrightarrow \mathrm{H} 5, \quad \mathrm{CCH}_{3} \quad$ (orthoester) $\leftrightarrow \mathrm{OCH}_{2} \mathrm{CH}_{3}$ (orthoester), and $\mathrm{H} 2 \leftrightarrow \mathrm{OCH}_{2} \mathrm{CH}_{3}$ (orthoester). From these results, it could be concluded that the rhamnopyranose ring of $\mathbf{2 0}$ is in the ${ }^{1} \mathrm{C}_{4}$ chair conformation, and in the 1,2-O-(1-ethoxyethylidene) derivative $\mathbf{2 0}$ the ethoxy group exists in an exo-orientation ( $S$-configuration). The distortion of the chair towards a half-chair conformation, as reported by Perlin, ${ }^{19,20}$ which is to be expected from the fusion of the five-membered orthoester group at positions 1 and 2 of the sugar molecule, was not observed for $\mathbf{2 0}$.

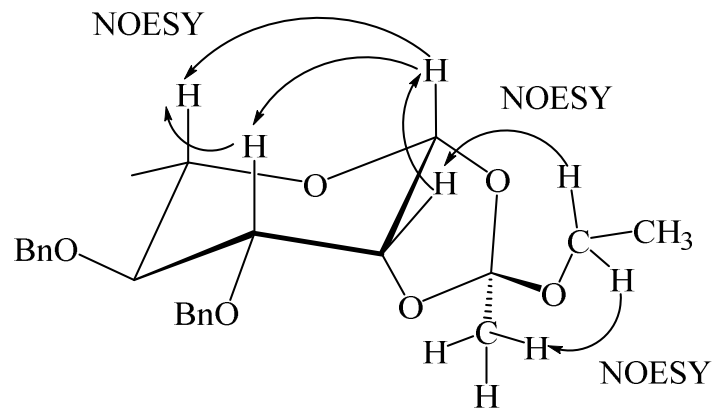

Figure 2. NOESY proton correlation of compound 20.

The X-ray diffraction studies of $\mathbf{2 0}$ (Figure 1) established the configuration at C-1, C-2 and provided information on the conformation of the pyranose ring. The puckering parameters Q: 0.549 (5) $\AA, \theta: 160.2(5)^{\circ}, \phi: 114.8(14)^{\circ}$ of the sugar ring, show a nearly ideal ${ }^{1} \mathrm{C}_{4}$ chair conformation.

Hydrolysis of $\mathbf{2 0}$ in $70 \% \mathrm{AcOH}$ solution followed by acetylation of the 1-position with acetic anhydride in presence of pyridine gave both $\alpha$ - and $\beta$-acetylated derivatives $21 \alpha$ and $21 \beta$ in $66 \%$ and $14 \%$ yields, respectively. ${ }^{1} \mathrm{H}$ NMR and NOESY experiments (Figure 3) confirm the configuration at the 1-position in the diacetates $21 \alpha$ and $21 \beta$. 

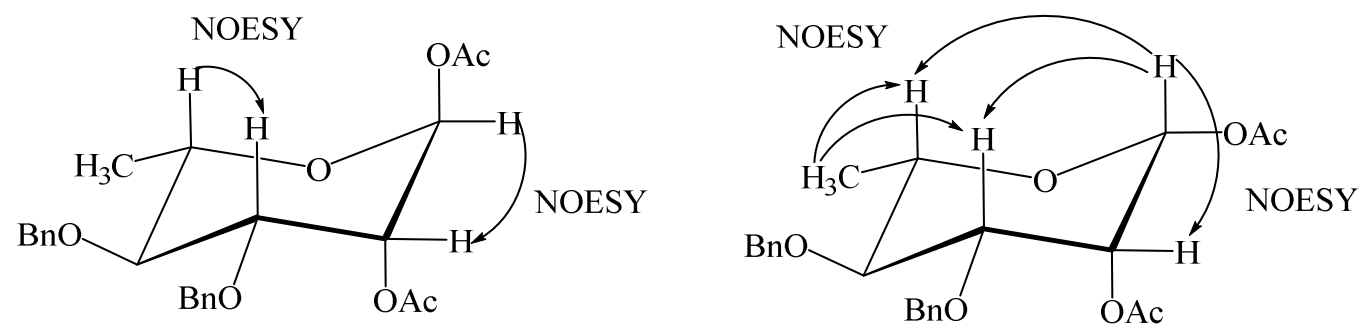

Figure 3. NOESY proton correlation of compounds $21 \alpha$ and $21 \beta$.

As shown in Scheme 5, the rhamnopyranose donors $21 \alpha \beta$ were coupled with the C-glycoside galacturonate acceptors 4 in a ratio of $1.1: 1$ in the presence of trimethylsilyl trifluoromethanesulfonate. ${ }^{10-12}$ This coupling produced the desired $\alpha$ - $(1 \rightarrow 4)$-coupled disaccharide $\mathbf{2 3}$ in $18 \%$ yield, $21 \alpha / \beta$ (donors) in $26 \%$ yield, and the accompanying transesterfication $^{21,22}$ product of the acceptor 25 in $23 \%$ yield. To improve the yield of the disaccharide 23 , the bromide donor 22 was synthesized from compounds $21 \alpha / \beta$ by the reaction with oxalyl bromide in dry dichloromethane to give the bromosugar 22 (Scheme 4) in 95\% yield. ${ }^{3,11}$ Compound $\mathbf{2 2}$ was unstable on storage and was used directly without further purification.

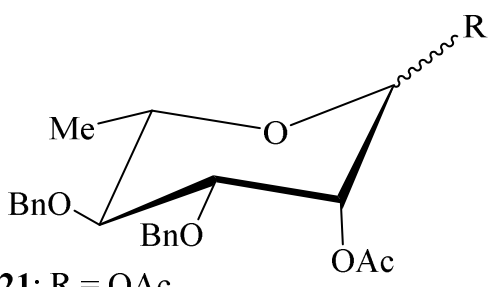

21: $\mathrm{R}=\mathrm{OAc}$

22: $\mathrm{R}=\mathrm{Br}$

$$
+
$$

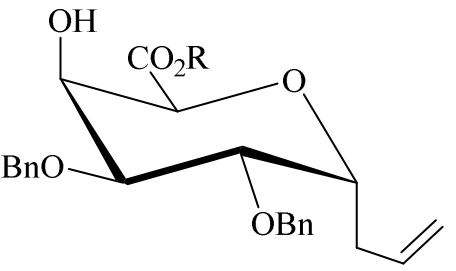

4: $\mathrm{R}=\mathrm{Me}$

5: $\mathrm{R}=\mathrm{Bn}$

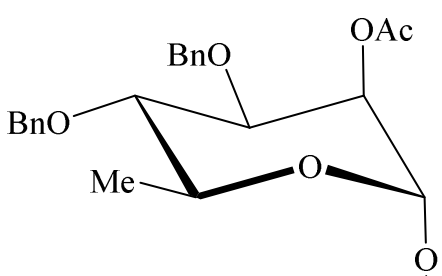

23: $\mathrm{R}=\mathrm{Bn}$

24: $\mathrm{R}=\mathrm{Me}$

$+$

$\mathrm{BnO}$

$\mathrm{CO}_{2} \mathrm{R}$
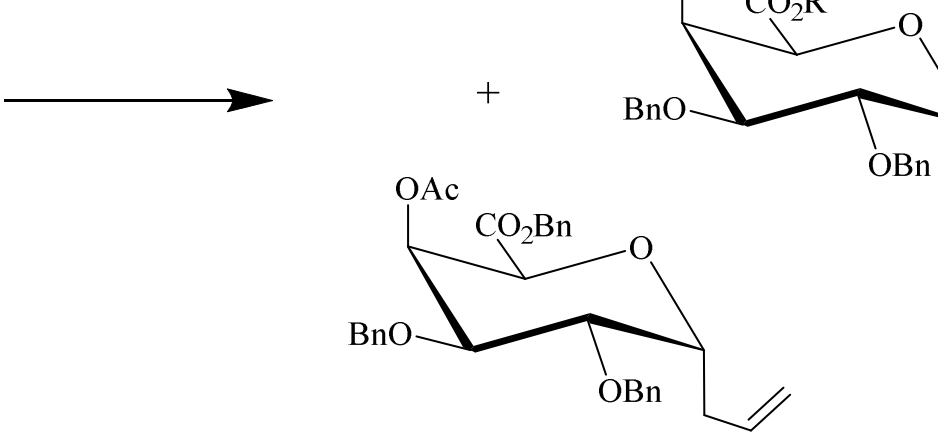

25

\section{Scheme 5}

The coupling of compounds 4 and 5 with 22 (Scheme 4) in the presence of 2,6-di-tert-butyl4-methylpyridine and silver trifluoromethanesulfonate ${ }^{3,11}$ for $2 \mathrm{~h}$ at $-70{ }^{\circ} \mathrm{C}$ gave the desired disaccharides 23 and 24 in 54\% and 56\% yields, respectively. The stereochemistry of the $O$ - 
glycosidic linkages were assigned based on the geminal coupling constants $J_{\mathrm{C}-1, \mathrm{H}-1}=170.46 \mathrm{~Hz}$ for disaccharide 23 and $J_{\mathrm{C}-1, \mathrm{H}-1}=171.5 \mathrm{~Hz}$ for disaccharide $24 .{ }^{3}$ In addition, all other analytical data of the disaccharides $\mathbf{2 3}$ and $\mathbf{2 4}$ supported the proposed structures.

\section{Conclusions}

Introduction of the trichloroacetimidate group at the anomeric center of $\mathbf{9}^{23,24}$ was reexamined, and the resulting trichloroacetimidate derivatives $\mathbf{1 3}$ were obtained in $98 \%$ total yield $(\alpha / \beta, 3: 1)$. Trichloroacetimidate derivatives $\mathbf{1 4}$ were synthesized in $76 \%$ total $(\alpha / \beta, 2: 1)$ yield and incorporated in our program as glycosyl donors.

The $(1 \rightarrow 4)$-linked disaccharide derivatives 15 were obtained in $53 \%$ total yield with low stereoselectivity $^{3,23,24}(\alpha, \beta 3: 1)$. The $\alpha$-linked disaccharide 15 and its $\beta$-linked anomer were successfully separated, and characterized by the aid of NMR spectroscopy. The change of the methyl ester to a benzyl ester in both glycosyl acceptor and donor had no strong influence on the outcome of the glycosylation. Disaccharide derivatives 16 were obtained in $44 \%$ total yield $(\alpha / \beta$, 2:1). Separation of $16 \alpha$ and $16 \beta$ was cumbersome and NMR investigation could be done only with enriched fractions.

Glycosyl derivatives $21 \alpha / \beta$ and the corresponding bromide ${ }^{25,26} \mathbf{2 2}$ were prepared as glycosyl donors in high yield from the orthoacetate 18. Glycosylation reaction of C-allylated galacturonate 5 with rhamnose acetates $21 \alpha, \beta$ gave only a moderate yield (18\%) compared with results for the glycosylation of $O$-allylated galacturonates ${ }^{3}$. In order to improve the yield, the more reactive bromide 22 was used. Glycosylation of acceptors $\mathbf{4}$ and $\mathbf{5}$ with $\mathbf{2 2}$ gave the $\alpha$ linked disaccharides 23 and $\mathbf{2 4}$ in 54\% and 56\% yields, respectively.

\section{Experimental Section}

General. Melting points were determined with a Boetius micro apparatus BHMK 05 (Rapido, Dresden) and are uncorrected. Optical rotations were measured for solutions in a 2-cm cell with an automatic polarimeter "Gyromat" (Dr. Kernchen Co.). Infrared analysis was recorded with an FT-IR spectrometer Nicolet (Protégé system 460). NMR spectra were recorded with Bruker Avance-500 spectrometers, (500.15 MHz for ${ }^{1} \mathrm{H}$, and $125.7 \mathrm{MHz}$ for ${ }^{13} \mathrm{C}$ ). The calibration of spectra was carried out by means of solvent peaks $\left(\mathrm{CDCl}_{3}: \delta{ }^{1} \mathrm{H} 7.25 \mathrm{ppm}, \delta{ }^{13} \mathrm{C} 77.00 \mathrm{ppm}\right.$; $\left.\mathrm{CD}_{3} \mathrm{OD}: \delta{ }^{1} \mathrm{H} 2.65 \mathrm{ppm}, \delta{ }^{13} \mathrm{C} 40.45 \mathrm{ppm}\right)$. Chemical shifts are given relative to the signal of internal standard tetramethylsilane $(\delta=0)$. First order chemical shifts and coupling constants were obtained from one-dimensional spectra, and assignment of proton resonance was based on COSY, COR, and NOSY experiments. For the determination of the molecular structure of compound 20 by X-ray diffraction, an X8Apex diffractometer system device was used with area detector and $\mathrm{Mo}-\mathrm{K}_{\alpha}$ radiation. A graphite monochromator system was used. The data collections 
were performed in routine $\omega$-scan at the Bruker P4 system, in $\omega / 2 \theta$-scans at the MACH3 and $\phi$ and $\omega$-scans at the area detector system. For the data collections on the four-circle diffractometer systems, suitable crystals were checked by rotational photos prior to the data collections. On the area detector system the crystal quality was evaluated by checking the reciprocal space plots after the collection of sixty frames. The structure solutions were obtained with the Bruker SHELXTL software. The refinement calculations were carried out by the full-matrix leastsquares method of SHELXL-97 (G.M.Sheldrick, SHELXL-97. programs for Crystal Structure Determinations, University of Göttingen, 1997). The non-hydrogen atoms were refined anisotropically while the hydrogen atoms were refined using the riding model. Copies of the data can be obtained free of charge on application to the Director, CCDC, 12 Union Road, Cambridge CB2 1RZ, UK (via e-mail: TUdeposit@ccdc.cam.ac.uk, or from the url address www.ccdc.cam.ac.uk/conts/retrieving.html)

Thin-layer chromatography (TLC) on pre-coated plates of silica gel (Merck Silica gel 60, FB254B, 0.25 mm) was performed with the following eluent systems (v/v): (A) 3:1, (B) 2:1, (C) 3:2, (D) 1:1, (E) 1:2 heptane-ethyl acetate, (F) 5:1, (G) 3:1 toluene-ethyl acetate, (H) 1:1:1:0.1 toluene-acetone-isopropanol-formic acid, (I) 4:2:2:1 toluene-ethyl acetate-ethanol-acetic acid, (J) 7:1, (K) 6:1 chloroform-methanol, (L) 1:1:0.05\% toluene-ethyl acetate-pyridine. The spots were made visible by spraying with methanolic $10 \% \mathrm{H}_{2} \mathrm{SO}_{4}$ solution and charring them for 3-5 min with a heat gun. Detection of benzyl derivatives was achieved by UV illumination. Preparative flash chromatography, MPLC and HPLC chromatography were performed by elution from columns of slurry-packed Silica Gel 60 (Merck, 40-63 $\mu \mathrm{m}$ ) and Nucleosil 100-7 (Knauer, 7.0 $\mu \mathrm{m})$, respectively. All solvents and reagents were purified and dried according to standard procedures $^{26}$. After classical work up of the reaction mixtures, the organic layers as a rule, were dried over $\mathrm{MgSO}_{4}$, and then concentrated under reduced pressure (rotary evaporator).

Benzyl 4- $\boldsymbol{O}$-acetyl-1- $\boldsymbol{O}$-allyl-2,3-di- $\boldsymbol{O}$-benzyl- $\boldsymbol{\beta}$-D-galactopyranuronate $(\mathbf{1 0})$. To a stirred solution of compound $\mathbf{8}(850 \mathrm{mg}, 1.68 \mathrm{mmol})$ in pyridine $(16.85 \mathrm{~mL})$ was added acetic anhydride $(8.42 \mathrm{~mL})$ at $0{ }^{\circ} \mathrm{C}$. After $3 \mathrm{~h}$ at ambient temperature (TLC eluent $\mathrm{D}, \mathrm{R}_{f} 0.67$ ), the mixture was poured into ice-water $(100 \mathrm{~mL})$. The aqueous layer was extracted with chloroform $(2 \times 45 \mathrm{~mL})$. The combined organic extracts were washed with cold aqueous $1 \%$ hydrochloric acid (35 mL), ice-water $(35 \mathrm{~mL})$, and cold saturated aqueous $\mathrm{NaHCO}_{3}(35 \mathrm{~mL})$, then dried and concentrated. The residue was purified by MPLC (eluent A) to give $\mathbf{1 0}(830 \mathrm{mg}, 98 \%)$ as colorless syrup: $[\alpha]_{\mathrm{D}}{ }^{21}+21.7$ (c 1.0, chloroform); ${ }^{1} \mathrm{H} \mathrm{NMR}\left(500 \mathrm{MHz}, \mathrm{CDCl}_{3}\right) \delta 1.97\left(\mathrm{OCOCH}_{3}\right), 3.63(\mathrm{dd}, 1 \mathrm{H}$, $\left.J_{3,4} 3.5 \mathrm{~Hz}, \mathrm{H}-3\right), 3.70$ (dd, 1H, $\left.J_{2,3} 9.8 \mathrm{~Hz}, \mathrm{H}-2\right), 4.18$ (d, 1H, $\left.J_{4,5} 1.2 \mathrm{~Hz}, \mathrm{H}-5\right), 4.17$ - 4.21 (m, $\left.2 \mathrm{H}, \mathrm{OCH}_{2} \mathrm{CHCH}_{2}\right), 4.48\left(\mathrm{~d}, 1 \mathrm{H}, J_{1,2} 7.8 \mathrm{~Hz}, \mathrm{H}-1\right), 4.55\left(\mathrm{~d}, 1 \mathrm{H}, J 11.5 \mathrm{~Hz}, \mathrm{OCH}_{2} \mathrm{C}_{6} \mathrm{H}_{5}\right), 4.74(\mathrm{~d}$, $\left.1 \mathrm{H}, J 11.0 \mathrm{~Hz}, \mathrm{OCH}_{2} \mathrm{C}_{6} \mathrm{H}_{5}\right), 4.78\left(\mathrm{~d}, 1 \mathrm{H}, J 11.5 \mathrm{~Hz}, \mathrm{OCH}_{2} \mathrm{C}_{6} \mathrm{H}_{5}\right), 4.92(\mathrm{~d}, 1 \mathrm{H}, J 11.0 \mathrm{~Hz}$, $\left.\mathrm{OCH}_{2} \mathrm{C}_{6} \mathrm{H}_{5}\right), 5.17\left(\mathrm{~d}, 1 \mathrm{H}, J 12.0 \mathrm{~Hz}, \mathrm{OCH}_{2} \mathrm{C}_{6} \mathrm{H}_{5}\right), 5.24\left(\mathrm{~d}, 1 \mathrm{H}, J 12.0 \mathrm{~Hz}, \mathrm{OCH}_{2} \mathrm{C}_{6} \mathrm{H}_{5}\right), 5.21-$ $5.25\left(\mathrm{~m}, 1 \mathrm{H}, \mathrm{OCH}_{2} \mathrm{CHCH}_{2}\right), 5.34$ - $5.39\left(\mathrm{~m}, 1 \mathrm{H}, \mathrm{OCH}_{2} \mathrm{CHCH}_{2}\right), 5.82$ (dd, $\left.1 \mathrm{H}, J_{4,5} 1.2 \mathrm{~Hz}, \mathrm{H}-4\right)$, $5.98\left(\mathrm{~m}, 1 \mathrm{H}, \mathrm{OCH}_{2} \mathrm{CHCH}_{2}\right), 7.25-7.44\left(\mathrm{~m}, 15 \mathrm{H}, \mathrm{OCH}_{2} \mathrm{C}_{6} \mathrm{H}_{5}\right) ;{ }^{13} \mathrm{C} \mathrm{NMR}\left(125.7 \mathrm{MHz}, \mathrm{CDCl}_{3}\right) \delta$ $20.53\left(\mathrm{COCH}_{3}\right), 67.39\left(\mathrm{CH}_{2} \mathrm{C}_{6} \mathrm{H}_{5}\right), 67.58(\mathrm{C}-4), 70.39\left(\mathrm{CH}_{2} \mathrm{C}_{6} \mathrm{H}_{5}\right), 72.23\left(\mathrm{CH}_{2} \mathrm{C}_{6} \mathrm{H}_{5}\right), 72.31(\mathrm{C}-$ 
5), $75.32\left(\mathrm{OCH}_{2} \mathrm{CHCH}_{2}\right), 78.17$ (C-2), 78.69 (C-3), $102.19(\mathrm{C}-1), 117.44\left(\mathrm{OCH}_{2} \mathrm{CH}=\mathrm{CH}_{2}\right)$, $127.53,127.65,127.93,128.00,128.15,128.32,128.51,128.54,128.92,134.89,137.72,138.34$ $\left(3 \times \mathrm{CH}_{2} \mathrm{C}_{6} \mathrm{H}_{5}\right.$ six signals are isochronous), $133.69\left(\mathrm{OCH}_{2} \mathrm{CH}=\mathrm{CH}_{2}\right), 166.53\left(\mathrm{CO}_{2} \mathrm{C}_{6} \mathrm{H}_{5}\right), 169.81$ $\left(\mathrm{COCH}_{3}\right)$. Anal. Calcd for $\mathrm{C}_{32} \mathrm{H}_{34} \mathrm{O}_{8}$ (546.61): C, 70.31, H, 6.27. Found: C, 69.54, H, 6.26.

Deallylation of compound 10. Benzyl 4- $O$-acetyl-2,3-di- $O$-benzyl- $\alpha$-D-galactopyranuronate (12). To a stirred solution of $\mathbf{1 0}(1.10 \mathrm{~g}, 1.9 \mathrm{mmol})$ in a mixture of acetic acid and water (20:1 $\mathrm{v} / \mathrm{v}, 58.50 \mathrm{~mL})$, anhydrous sodium acetate $(1.6 \mathrm{~g}, 1.95 \mathrm{mmol})$ and palladium(II) chloride $(1.38 \mathrm{~g}$, $7.77 \mathrm{mmol}$ ) were added. After stirring for $4 \mathrm{~h}$ at $40-45^{\circ} \mathrm{C}$ (TLC eluent $\mathrm{F}, \mathrm{R}_{f} 0.48$ ), the reaction mixture was filtered, and the solids were washed with chloroform. The combined filtrate and washings were washed with sat aq $\mathrm{NaHCO}_{3}(3 \times 50 \mathrm{~mL})$, and water $(2 \times 50 \mathrm{~mL})$, and were dried and concentrated. The residue was purified by flash chromatography (eluent $\mathrm{F}$ ) to provide $\mathbf{1 2}$ $(610 \mathrm{mg}, 56 \%)$ as an amorphous yellow solid: $[\alpha]_{\mathrm{D}}{ }^{21}+29.7$ (c 1.0, chloroform); ${ }^{1} \mathrm{H}$ NMR (500 $\mathrm{MHz}_{\mathrm{CDCl}}$ ): $\delta 1.89$ (s, 3H, $\mathrm{COCH}_{3}$ ), 3.12 (br., $1 \mathrm{H}, \mathrm{OH}$ ), 3.79 (dd, $1 \mathrm{H} J_{2,3} 9.8 \mathrm{~Hz}, \mathrm{H}-2$ ), 3.98 $\left(\mathrm{dd}, 1 \mathrm{H}, J_{3,4} 3.5 \mathrm{~Hz}, \mathrm{H}-3\right), 4.53$ (d, $\left.1 \mathrm{H}, J 11.0 \mathrm{~Hz}, \mathrm{CH}_{2} \mathrm{C}_{6} \mathrm{H}_{5}\right), 4.64$ (d, $1 \mathrm{H}, J 11.7 \mathrm{~Hz}, \mathrm{CH}_{2} \mathrm{C}_{6} \mathrm{H}_{5}$ ), $4.77\left(\mathrm{~d}, 1 \mathrm{H}, J 11.0 \mathrm{~Hz}, \mathrm{CH}_{2} \mathrm{C}_{6} \mathrm{H}_{5}\right), 4.78(\mathrm{~d}, 1 \mathrm{H}, J 1.5 \mathrm{~Hz}, \mathrm{H}-5), 4.81(\mathrm{~d}, 1 \mathrm{H}, J 11.7 \mathrm{~Hz}$, $\mathrm{CH}_{2} \mathrm{C}_{6} \mathrm{H}_{5}$ ), 5.20 (center of AB, $2 \mathrm{H}, J 12.0 \mathrm{~Hz}, \mathrm{CH}_{2} \mathrm{C}_{6} \mathrm{H}_{5}$ ), $5.40\left(\mathrm{~d}, 1 \mathrm{H}, J_{1,2} 3.5 \mathrm{~Hz}, \mathrm{H}-1\right.$ ), 5.90 (dd, $\left.1 \mathrm{H}, J_{4,5} 1.5 \mathrm{~Hz}, \mathrm{H}-4\right), 7.40-7.25\left(\mathrm{~m}, 15 \mathrm{H}, \mathrm{C}_{6} H_{5}\right) ;{ }^{13} \mathrm{C} \mathrm{NMR}\left(125.7 \mathrm{MHz}, \mathrm{CDCl}_{3}\right): \delta 20.54$ $\left(\mathrm{OCOCH}_{3}\right), \quad 67.49 \quad\left(\mathrm{OCH}_{2} \mathrm{C}_{6} \mathrm{H}_{5}\right), 68.43 \quad(\mathrm{C}-4), 69.02 \quad(\mathrm{C}-5), \quad 72.17 \quad\left(\mathrm{OCH}_{2} \mathrm{C}_{6} \mathrm{H}_{5}\right), \quad 73.89$ $\left(\mathrm{OCH}_{2} \mathrm{C}_{6} \mathrm{H}_{5}\right), 74.94(\mathrm{C}-2), 75.40$ (C-3), 92.14 (C-1), 127.75, 127.95, 127.98, 128.03, 128.37, 128.64, 129.10, 134.96, 137.73, $137.91\left(\mathrm{OCH}_{2} \mathrm{C}_{6} \mathrm{H}_{5}\right.$ eight signals are isochronous), 167.69 $\left(\mathrm{CO}_{2} \mathrm{CH}_{2} \mathrm{C}_{6} \mathrm{H}_{5}\right), 169.76\left(\mathrm{OCOCH}_{3}\right)$. Anal. Calcd for $\mathrm{C}_{29} \mathrm{H}_{30} \mathrm{O}_{8}(506.54)$ : C, 68.76, H, 5.97. Found: C, 68.82, H, 6.03.

Introduction of the trichloroacetamidate group at the anomeric center of 11 . To a solution of compound $11(330 \mathrm{mg}, 0.77 \mathrm{mmol})$ in dry dichloromethane $(5 \mathrm{~mL})$, trichloroacetonitrile $(2.84$ $\mathrm{mL}, 28.4 \mathrm{mmol}$ ) and 1,8-diazabicyclo[5.4.0]undec-7-ene (DBU, $23 \mu \mathrm{L}, 0.15 \mathrm{mmol}$ ) were added under argon at $-20{ }^{\circ} \mathrm{C}$. The reaction mixture was stirred at that temperature for one hour, and then for an additional hour at room temperature (TLC eluent $B ; R_{f} .0 .39, R_{f}$ 0.27). Finally, the mixture was concentrated. The residue was suspended in ethyl acetate $(30 \mathrm{~mL})$ and filtered over a layer of silica gel. The remaining solids were washed with ethyl acetate $(3 \times 8 \mathrm{~mL})$; the combined filtrate and washings were dried and concentrated. The residue was purified by HPLC (eluent ethyl acetate gradient $0 \% \rightarrow 50 \%$ in petrol ether $\mathrm{v} / \mathrm{v}$ ) to provide the product.

Methyl 4- $\boldsymbol{O}$-acetyl-2,3-di- $\boldsymbol{O}$-benzyl-1-(trichloroacetimidoyloxy)- $\alpha$-D-galactopyranosyluronate $(\mathbf{1 3 \alpha})\left(320 \mathrm{mg}, 73 \%, \mathrm{R}_{f} 0.39\right)$, yellow syrup; $[\alpha]_{\mathrm{D}}{ }^{22}+88.9$ (c 1.4, chloroform); ${ }^{1} \mathrm{H} \mathrm{NMR}$ $\left(500 \mathrm{MHz}, \mathrm{CDCl}_{3}\right) \delta 2.09\left(\mathrm{~s}, 3 \mathrm{H}, \mathrm{COCH}_{3}\right), 3.74\left(\mathrm{~s}, 3 \mathrm{H}, \mathrm{CO}_{2} \mathrm{CH}_{3}\right), 4.04\left(\mathrm{dd}, 1 \mathrm{H}, J_{2,3} 10.0 \mathrm{~Hz}, \mathrm{H}-\right.$ 2), 4.09 (dd, $\left.J_{3,4} 3.5 \mathrm{~Hz}, \mathrm{H}-3\right), 4.61$ (d, $\left.1 \mathrm{H}, J 11.8 \mathrm{~Hz}, \mathrm{CH}_{2} \mathrm{C}_{6} \mathrm{H}_{5}\right), 4.71$ (d, 1H, J $\left.1.5 \mathrm{~Hz}, \mathrm{H}-5\right)$, 4.74 (center of $\left.\mathrm{AB}, 2 \mathrm{H}, \mathrm{CH}_{2} \mathrm{C}_{6} \mathrm{H}_{5}\right), 4.78\left(\mathrm{~d}, 2 \mathrm{H}, J 10.8 \mathrm{~Hz}, \mathrm{CH}_{2} \mathrm{C}_{6} \mathrm{H}_{5}\right), 5.90$ (dd, $1 \mathrm{H}, J_{4,5} 1.5 \mathrm{~Hz}$, $\mathrm{H}-4), 6.69\left(\mathrm{~d}, 1 \mathrm{H}, J_{1,2} 3.5 \mathrm{~Hz}, \mathrm{H}-1\right), 7.22-7.35\left(\mathrm{~m}, 15 \mathrm{H}, \mathrm{C}_{6} H_{5}\right), 8.66$ [s, $\left.1 \mathrm{H}, \mathrm{O}(\mathrm{C}=\mathrm{N} H) \mathrm{CCl}_{3}\right]$; ${ }^{13} \mathrm{C}$ NMR $\left(125.7 \mathrm{MHz}, \mathrm{CDCl}_{3}\right) \delta 20.55\left(\mathrm{OCOCH}_{3}\right), 52.54\left(\mathrm{CO}_{2} \mathrm{CH}_{3}\right), 68.23(\mathrm{C}-4), 71.12(\mathrm{C}-5)$, $72.02\left(\mathrm{OCH}_{2} \mathrm{C}_{6} \mathrm{H}_{5}\right), 73.14\left(\mathrm{OCH}_{2} \mathrm{C}_{6} \mathrm{H}_{5}\right), 74.19(\mathrm{C}-2), 74.38(\mathrm{C}-3), 90.91$ [OC(=NH)CCl 3 ], 94.38 
(C-1), 127.33, 127.51, 127.95, 128.15, 128.21, 137.42, $137.92\left(2 \times \mathrm{OCH}_{2} C_{6} \mathrm{H}_{5}\right.$ five signals are isochronous), $160.43\left[\mathrm{OC}(=\mathrm{NH}) \mathrm{CCl}_{3}\right], 167.17\left(\mathrm{CO}_{2} \mathrm{Me}\right), 169.89\left(\mathrm{OCOCH}_{3}\right)$.

Methyl 4- $\boldsymbol{O}$-acetyl-2,3-di- $\boldsymbol{O}$-benzyl-1-(trichloroacetimidoyloxy)- $\boldsymbol{\beta}$-D-galactopyranosyluronate $(13 \beta)\left(110 \mathrm{mg}, 25 \%, \mathrm{R}_{f} 0.27\right)$, yellow color syrup; $[\alpha] \mathrm{D}^{22}+67.1(c 1.05$, chloroform); ${ }^{1} \mathrm{H}$ NMR $\left(500 \mathrm{MHz}, \mathrm{CDCl}_{3}\right) \delta 2.14\left(\mathrm{~s}, \mathrm{COCH}_{3}\right), 3.75\left(\mathrm{dd}, 1 \mathrm{H}, J_{3,4} 3.5 \mathrm{~Hz}, \mathrm{H}-3\right), 3.75(\mathrm{~s}, 3 \mathrm{H}$, $\mathrm{CO}_{2} \mathrm{CH}_{3}$ ), 3.91(dd, $\left.1 \mathrm{H}, J_{1,2} 8.2 \mathrm{~Hz}, \mathrm{H}-2\right), 4.35$ (d, $\left.1 \mathrm{H}, J_{4,5} 1.5 \mathrm{~Hz}, \mathrm{H}-5\right), 4.55$ (d, 1H, J 11.5 Hz, $\left.\mathrm{OCH}_{2} \mathrm{C}_{6} \mathrm{H}_{5}\right), 4.78\left(\mathrm{~d}, 2 \mathrm{H}, J 11.5 \mathrm{~Hz}, \mathrm{OCH}_{2} \mathrm{C}_{6} \mathrm{H}_{5}\right), 4.79\left(\mathrm{~d}, 2 \mathrm{H}, J 10.8 \mathrm{~Hz}, \mathrm{OCH}_{2} \mathrm{C}_{6} \mathrm{H}_{5}\right), 4.88(\mathrm{~d}$, $\left.1 \mathrm{H}, J 10.8 \mathrm{~Hz}, \mathrm{OCH}_{2} \mathrm{C}_{6} \mathrm{H}_{5}\right), 5.79\left(\mathrm{~d}, 1 \mathrm{H}, J_{1,2} 8.2 \mathrm{~Hz}, \mathrm{H}-1\right), 5.84\left(\mathrm{dd}, 1 \mathrm{H}, J_{3,4} 3.5 \mathrm{~Hz}, J_{4,5} 1.5 \mathrm{~Hz}\right.$, $\mathrm{H}-4), 7.24-7.34\left(\mathrm{~m}, 15 \mathrm{H}, \mathrm{C}_{6} H_{5}\right), 8.73\left[\mathrm{~s}, 1 \mathrm{H},(\mathrm{C}=\mathrm{NH}) \mathrm{CCl}_{3}\right] .{ }^{13} \mathrm{C} \mathrm{NMR}\left(125.7 \mathrm{MHz}, \mathrm{CDCl}_{3}\right)$ $\delta 20.55\left(\mathrm{OCOCH}_{3}\right), 52.50\left(\mathrm{CO}_{2} \mathrm{CH}_{3}\right), 67.25(\mathrm{C}-4), 72.10\left(\mathrm{OCH}_{2} \mathrm{C}_{6} \mathrm{H}_{5}\right), 73.00(\mathrm{C}-5), 75.15$ $\left(\mathrm{OCH}_{2} \mathrm{C}_{6} \mathrm{H}_{5}\right), 76.61(\mathrm{C}-2), 78.62(\mathrm{C}-3), 91.92$ [OC(=NH)CCl 3$], 97.52(\mathrm{C}-1), 127.55,127.77$, 128.18, 128.22, 137.26, $137.67\left(2 \times \mathrm{OCH}_{2} \mathrm{C}_{6} \mathrm{H}_{5}\right.$ six signals are isochronous $), 163.41$ $\left[\mathrm{OC}(=\mathrm{NH}) \mathrm{CCl}_{3}\right], 168.26\left(\mathrm{CO}_{2} \mathrm{CH}_{3}\right), 169.83\left(\mathrm{OCOCH}_{3}\right)$.

Introduction of the trichloroacetimidate group at the anomeric center of 12. To a solution of compound $12(510 \mathrm{mg}, 1.0 \mathrm{mmol})$ in dry dichloromethane $(7 \mathrm{ml})$, trichloroacetonitrile $(3.7 \mathrm{~mL}$, $37 \mathrm{mmol}$ ) and 1,8-diazabicyclo[5.4.0]undec-7-ene (DBU $30 \mu \mathrm{l}, 0.20 \mathrm{mmol}$ ) were added under argon at $-20^{\circ} \mathrm{C}$. The reaction mixture was stirred at that temperature for one hour, and then for an additional hour at room temperature (TLC eluent F; $\mathrm{R}_{f .} 0.77, \mathrm{R}_{f} .0 .73$ ). Finally, the mixture was concentrated; the residue was suspended in ethyl acetate $(30 \mathrm{~mL})$ and filtered over a layer of silica gel. The remaining solids were washed with ethyl acetate $(3 \times 8 \mathrm{~mL})$, the combined filtrate and washings were dried and concentrated. The residue was purified by HPLC (eluent ethyl acetate gradient $0 \% \rightarrow 50 \%$ in petrol ether v/v) to provide $14 \alpha\left(\mathrm{R}_{f} 0.77,210 \mathrm{mg}, 40 \%\right), 14 \beta\left(\mathrm{R}_{f}\right.$ $0.73,100 \mathrm{mg}, 19 \%$ ), and $\alpha / \beta$ mixture (90 mg, 17\%).

Benzyl 4- $\boldsymbol{O}$-acetyl-2,3-di- $\boldsymbol{O}$-benzyl-1-(trichloroacetimidoyloxy)- $\alpha$-D-galactopyranosyluronate $(14 \alpha):[\alpha]_{\mathrm{D}}{ }^{21}+70.0$ (c 1.0, chloroform); $1 \mathrm{H} \mathrm{NMR}\left(500 \mathrm{MHz}, \mathrm{CDCl}_{3}\right) \delta 1.87(\mathrm{~s}, 3 \mathrm{H}$, $\mathrm{COCH}_{3}$ ), 3.97 (dd, 1H, J $\left.J_{2,3} 10.0 \mathrm{~Hz}, \mathrm{H}-2\right), 4.03$ (dd, 1H, $\left.J_{3,4} 2.1 \mathrm{~Hz}, \mathrm{H}-3\right), 4.54$ (d, 1H, J 11.5 $\mathrm{Hz}, \mathrm{CH}_{2} \mathrm{C}_{6} \mathrm{H}_{5}$ ), 4.69 (center of $\left.\mathrm{AB}, 2 \mathrm{H}, J_{\mathrm{A}, \mathrm{B}} 11.8 \mathrm{~Hz}, \mathrm{CH}_{2} \mathrm{C}_{6} \mathrm{H}_{5}\right), 4.70\left(\mathrm{~d}, 1 \mathrm{H}, J_{4,5} 1.5 \mathrm{~Hz}, \mathrm{H}-5\right.$ ), $4.72\left(\mathrm{~d}, 1 \mathrm{H}, J 11.5 \mathrm{~Hz}, \mathrm{CH}_{2} \mathrm{C}_{6} \mathrm{H}_{5}\right.$ ), 5.12 (center of $\mathrm{AB}, 2 \mathrm{H}, J_{\mathrm{A}, \mathrm{B}} 11.8 \mathrm{~Hz}, \mathrm{CH}_{2} \mathrm{C}_{6} \mathrm{H}_{5}$ ), 5.87 (dd, $\left.1 \mathrm{H}, J_{4,5} 1.5 \mathrm{~Hz}, \mathrm{H}-4\right), 6.65\left(\mathrm{~d}, 1 \mathrm{H}, J_{1,2} 3.5 \mathrm{~Hz}, \mathrm{H}-1\right), 7.21-7.36\left(\mathrm{~m}, 15 \mathrm{H}, \mathrm{C}_{6} H_{5}\right), 8.60[\mathrm{~s}, 1 \mathrm{H}$, $\left.(\mathrm{C}=\mathrm{NH}) \mathrm{CCl}_{3}\right],{ }^{13} \mathrm{C} \mathrm{NMR}\left(500 \mathrm{MHz}, \mathrm{CDCl}_{3}\right) \delta 20.46\left(\mathrm{COCH}_{3}\right), 67.72\left(\mathrm{CH}_{2} \mathrm{C}_{6} \mathrm{H}_{5}\right), 68.23(\mathrm{C}-4)$, 71.20 (C-5), $72.13 \quad\left(\mathrm{CH}_{2} \mathrm{C}_{6} \mathrm{H}_{5}\right), \quad 73.23 \quad\left(\mathrm{CH}_{2} \mathrm{C}_{6} \mathrm{H}_{5}\right), 74.27 \quad(\mathrm{C}-2), \quad 74.53 \quad(\mathrm{C}-3), 91.01$ $\left[\mathrm{O}(\mathrm{C}=\mathrm{NH}) \mathrm{CCl}_{3}\right], 94.54(\mathrm{C}-1), 127.41,127.57,127.69,128.06,128.22,128.27,128.64,128.72$, $129.16,134.75,137.53,138.03\left(3 \times \mathrm{OCH}_{2} \mathrm{C}_{6} \mathrm{H}_{5}\right.$ six signals are isochronous $), 160.59$ $\left[\mathrm{O}(C=\mathrm{NH}) \mathrm{CCl}_{3}\right], 166.69 \quad\left(\mathrm{CO}_{2} \mathrm{CH}_{2} \mathrm{C}_{6} \mathrm{H}_{5}\right), 169.62\left(\mathrm{OCOCH}_{3}\right) ; \quad \mathrm{FAB} \quad[\mathrm{M}+\mathrm{Na}]+: \mathrm{m} / z \quad 674$. Anal.Calcd for $\mathrm{C}_{29} \mathrm{H}_{30} \mathrm{O}_{8}$ (650.93): C, 57.20, H, 4.65, N, 2.15 Found: C, 57.64, H, 4.69, N, 2.10;

Benzyl 4- $\boldsymbol{O}$-acetyl-2,3-di- $\boldsymbol{O}$-benzyl-1-(trichloroacetimidoyloxy)- $\boldsymbol{\beta}$-D-galactopyranosyluronate $(14 \beta):[\alpha]_{\mathrm{D}}{ }^{21}+44.6$ (c 1.0, chloroform); $1 \mathrm{H} \mathrm{NMR}\left(500 \mathrm{MHz}, \mathrm{CDCl}_{3}\right) \delta 1.95(\mathrm{~s}, 3 \mathrm{H}$, $\mathrm{COCH}_{3}$ ), 3.73 (dd, $1 \mathrm{H}, J_{3,4} 3.5 \mathrm{~Hz}, \mathrm{H}-3$ ), 3.88 (dd, 1H, $\left.J_{2,3} 9.8 \mathrm{~Hz}, \mathrm{H}-2\right), 4.37$ (d, 1H, J $1.5 \mathrm{~Hz}$, $\mathrm{H}-5), 4.52\left(\mathrm{~d}, 1 \mathrm{H}, J 11.5 \mathrm{~Hz}, \mathrm{CH}_{2} \mathrm{C}_{6} \mathrm{H}_{5}\right), 4.76\left(\mathrm{~d}, 1 \mathrm{H}, J 10.7 \mathrm{~Hz}, \mathrm{CH}_{2} \mathrm{C}_{6} \mathrm{H}_{5}\right), 4.77(\mathrm{~d}, 1 \mathrm{H}, J 11.5$ $\mathrm{Hz}, \mathrm{CH}_{2} \mathrm{C}_{6} \mathrm{H}_{5}$ ), $4.87\left(\mathrm{~d}, 1 \mathrm{H}, J 10.7 \mathrm{~Hz}, \mathrm{CH}_{2} \mathrm{C}_{6} \mathrm{H}_{5}\right.$ ), 5.16 (center of $\mathrm{AB}, 2 \mathrm{H}, J_{\mathrm{A}, \mathrm{B}} 12.0 \mathrm{~Hz}$, 
$\left.\mathrm{CH}_{2} \mathrm{C}_{6} \mathrm{H}_{5}\right), 5.79\left(\mathrm{~d}, 1 \mathrm{H}, J_{1,2} 8.2 \mathrm{~Hz}, \mathrm{H}-1\right), 5.85$ (dd, $\left.1 \mathrm{H}, J_{3,4} 3.5 \mathrm{~Hz}, J_{4,5} 1.5 \mathrm{~Hz}, \mathrm{H}-4\right), 7.24-7.40$ $\left(\mathrm{m}, 15 \mathrm{H}, \mathrm{C}_{6} \mathrm{H}_{5}\right), 8.72\left[\mathrm{~s}, 1 \mathrm{H},(\mathrm{C}=\mathrm{NH}) \mathrm{CCl}_{3}\right],{ }^{13} \mathrm{C} \mathrm{NMR}\left(500 \mathrm{MHz}, \mathrm{CDCl}_{3}\right) \delta 20.59\left(\mathrm{COCH}_{3}\right)$, 67.40 (C-4), $72.48\left(\mathrm{CH}_{2} \mathrm{C}_{6} \mathrm{H}_{5}\right), 73.17$ (C-5), $75.41\left(\mathrm{CH}_{2} \mathrm{C}_{6} \mathrm{H}_{5}\right), 76.88$ (C-2), 79.03 (C-3), 90.73 $\left[(\mathrm{C}=\mathrm{NH}) \mathrm{CCl}_{3}\right], 97.78(\mathrm{C}-1), 127.71,127.87,127.90,128.11,128.25,128.38,128.60,128.64$, 129.04, 134.89, 137.34, $137.92\left(3 \times \mathrm{CH}_{2} \mathrm{C}_{6} \mathrm{H}_{5}\right.$ three signals are isochronous $), 161.27$ $\left[(C=\mathrm{NH}) \mathrm{CCl}_{3}\right], 165.83\left(\mathrm{CO}_{2} \mathrm{CH}_{2} \mathrm{C}_{6} \mathrm{H}_{5}\right), 169.80\left(\mathrm{OCOCH}_{3}\right)$.

Glycosylation reactions of D-galacturonate acceptors with D-galacturonate donors. A mixture of glycosyl acceptor 4 (170 mg, $0.4 \mathrm{mmol}$ ), glycosyl donors $\mathbf{1 2} \alpha / \beta$ (290 mg, $0.5 \mathrm{mmol}$ ), and powdered activated molecular sieves $(4 \AA, 4.0 \mathrm{~g})$ was dried azeotropically with toluene, and then subjected to high vacuum for $2 \mathrm{~h}$. The mixture was dissolved in dry dichloromethane ( 8 $\mathrm{mL}$ ), and stirred for $2 \mathrm{~h}$ under argon at room temperature. After cooling to $-70{ }^{\circ} \mathrm{C}$, TMSOTf (83 $\mu \mathrm{L}, 0.5 \mathrm{mmol}$ ) was added, and stirring was continued for $3 \mathrm{~h}$ at that temperature. The reaction mixture was then allowed to warm-up to room temperature and stirring was continued for an additional $18 \mathrm{~h}$ (TLC eluent $\mathrm{D} ; \mathrm{R}_{f .} 0.35, \mathrm{R}_{f .}$ 0.33). The reaction mixture was passed through a layer of alkaline alumina by elution with chloroform. The eluate was concentrated to $(30 \mathrm{~mL})$ then was washed with cold aq sat $\mathrm{NaHCO}_{3}(2 \times 15 \mathrm{~mL})$, and ice-water $(2 \times 15 \mathrm{~mL})$, dried and concentrated. The crude disaccharides were purified by HPLC (eluent ethyl acetate gradient $0 \%$ $\rightarrow 25 \%$ in petrol ether v/v) to yield $15 \alpha / \beta(180 \mathrm{mg}, 53 \%)$ in the ratio $3: 1$.

Methyl (methyl 4-O-acetyl-2,3-di- $O$-benzyl- $\alpha$-D-galactopyranosyluronate)-(1 $\rightarrow$ 4)-2,3-di- $O$ benzyl-1-deoxy-1-(prop-2-enyl)- $\alpha$-D-galactopyranuronate $(\mathbf{1 5} \alpha) .(120 \mathrm{mg}, 35 \%){ }^{1} \mathrm{H}$ NMR $\left(500 \mathrm{MHz}, \mathrm{CDCl}_{3}\right) \delta 2.02\left(\mathrm{~s}, 3 \mathrm{H}, \mathrm{OCOCH}_{3}\right), 2.27-2.48\left(\mathrm{~m}, 2 \mathrm{H},-\mathrm{CH}_{2} \mathrm{CHCH}_{2}\right), 3.47,3.51(2 \times \mathrm{s}$, $6 \mathrm{H}, \mathrm{CO}_{2} \mathrm{CH}_{3}$ ), 3.58-3.62 (m, 1H, H-2), 3.79-3.85 (m, 3H, H-2', H-3', H-3), 3.97 (dd, 1H, J3,4 10.5 $\left.\mathrm{Hz}, J_{4,5} 3.5 \mathrm{~Hz}, \mathrm{H}-4\right), 4.24$ (ddd, $\left.1 \mathrm{H}, J_{1,2} 2.5 \mathrm{~Hz}, J_{1, \mathrm{Ha}} 6.0 \mathrm{~Hz}, J_{1, \mathrm{Hb}} 8.5 \mathrm{~Hz}, \mathrm{H}-1\right), 4.52-4.76$ (m, $\left.9 \mathrm{H}, \mathrm{H}-4{ }^{\prime}, \mathrm{CH}_{2} \mathrm{C}_{6} \mathrm{H}_{5}\right), 4.83\left(\mathrm{~d}, 1 \mathrm{H}, \mathrm{H}-5^{\prime}\right), 5.04-5.14\left(4 \times \mathrm{m}, 2 \mathrm{H},-\mathrm{CH}_{2} \mathrm{CHCH}_{2}\right), 5.16\left(\mathrm{~d}, 1 \mathrm{H}, J_{1^{\prime}, 2^{\prime}}\right.$ $\left.3.5 \mathrm{~Hz}, \mathrm{H}-1^{\prime}\right), 5.75-5.85\left(\mathrm{~m}, 1 \mathrm{H},-\mathrm{CH}_{2} \mathrm{CHCH}_{2}\right), 7.20-7.36\left(\mathrm{~m}, 20 \mathrm{H},-\mathrm{CH}_{2} \mathrm{C}_{6} \mathrm{H}_{5}\right) ;{ }^{13} \mathrm{C} \mathrm{NMR}(125.7$ $\left.\mathrm{MHz}, \mathrm{CDCl}_{3}\right) \delta 20.64\left(\mathrm{OCOCH}_{3}\right), 31.98\left(-\mathrm{CH}_{2} \mathrm{CHCH}_{2}\right), 51.9,52.2\left(2 \times \mathrm{CO}_{2} \mathrm{CH}_{3}\right), 68.86,69.79$, $70.89,71.30,71.40,72.99,73.16,73.22,73.41,75.27,75.30,75.5,76.28\left[\left(4 \times \mathrm{CH}_{2} \mathrm{C}_{6} \mathrm{H}_{5}\right),\left(\mathrm{C} 2^{\prime}-\right.\right.$ C5), (C1-C5)], 98.80 (C-1'), $117.00\left(-\mathrm{CH}_{2} \mathrm{CH}=\mathrm{CH}_{2}\right), 134.5\left(-\mathrm{CH}_{2} \mathrm{CHCH}_{2}\right), 137.68-128.45$ $\left(\mathrm{CH}_{2} \mathrm{C}_{6} \mathrm{H}_{5}\right), 169.70,169.80,171.90\left(3 \times \mathrm{COCH}_{3}\right) ; \mathrm{FAB}[\mathrm{M}+\mathrm{Na}]^{+}: \mathrm{m} / z 847$.

Methyl (methyl 4-O-acetyl-2,3-di- $O$-benzyl- $\beta$-D-galactopyranosyluronate)-(1 $\rightarrow$ 4)-2,3-di- $O$ benzyl-1-deoxy-1-(prop-2-enyl)- $\alpha$-D-galactopyranuronate $(15 \beta)$. (60 mg, 18\%) ${ }^{1} \mathrm{H}$ NMR (500 $\left.\mathrm{MHz}, \mathrm{CDCl}_{3}\right) \delta 2.07\left(\mathrm{~s}, 3 \mathrm{H}, \mathrm{OCOCH}_{3}\right), 2.39\left(\mathrm{~m}, 2 \mathrm{H},-\mathrm{CH}_{2} \mathrm{CHCH}_{2}\right), 3.51-3.64(\mathrm{~m}, 3 \mathrm{H}, \mathrm{H}-2$ ' , H3', H-4) , 3.74, 3.78 (2 × s, 6H, $\left.\mathrm{CO}_{2} \mathrm{CH}_{3}\right), 3.73-3.85$ (m, 2H, H-2, H-3), 4.41 (m, 1H, H-1), 4.36 $\left(\mathrm{d}, 1 \mathrm{H}, J 11.7 \mathrm{~Hz}, \mathrm{CH}_{2} \mathrm{C}_{6} \mathrm{H}_{5}\right), 4.44\left(\mathrm{~d}, 1 \mathrm{H}, J 11.7 \mathrm{~Hz}, \mathrm{CH}_{2} \mathrm{C}_{6} \mathrm{H}_{5}\right), 4.46\left(\mathrm{~d}, 1 \mathrm{H}, J_{4,5} 3.5 \mathrm{~Hz}, \mathrm{H}-5\right)$, $4.52\left(\mathrm{~d}, 1 \mathrm{H}, J 11.7 \mathrm{~Hz}, \mathrm{CH}_{2} \mathrm{C}_{6} \mathrm{H}_{5}\right.$ ), 4.36 (center of $\mathrm{AB}, 2 \mathrm{H}, J_{\mathrm{A}, \mathrm{B}} 12 \mathrm{~Hz}, \mathrm{CH}_{2} \mathrm{C}_{6} \mathrm{H}_{5}$ ), 4.66-4.73 (m, 2H, H-1', H-4), 4.73 (d, 1H, J $11.7 \mathrm{~Hz}, \mathrm{CH}_{2} \mathrm{C}_{6} \mathrm{H}_{5}$ ), 4.77 (d, $1 \mathrm{H}, J 11.5 \mathrm{~Hz}, \mathrm{CH}_{2} \mathrm{C}_{6} \mathrm{H}_{5}$ ), 4.97 (d, $\left.1 \mathrm{H}, J 11.5 \mathrm{~Hz}, \mathrm{CH}_{2} \mathrm{C}_{6} \mathrm{H}_{5}\right), 5.04-5.12\left(4 \times \mathrm{m}, 2 \mathrm{H},-\mathrm{CH}_{2} \mathrm{CHCH}_{2}\right), 5.70\left(\mathrm{~d}, 1 \mathrm{H}, \mathrm{H}-5^{\prime}\right), 5.81(\mathrm{~m}, 1 \mathrm{H}$, $\left.-\mathrm{CH}_{2} \mathrm{CHCH}_{2}\right)$, 7.16-7.35 (m, 20H, $\left.-\mathrm{CH}_{2} \mathrm{C}_{6} \mathrm{H}_{5}\right) ;{ }^{13} \mathrm{C} \mathrm{NMR}\left(125.7 \mathrm{MHz}, \mathrm{CDCl}_{3}\right) \delta 20.72$ $\left(\mathrm{OCOCH}_{3}\right), 32.04\left(-\mathrm{CH}_{2} \mathrm{CHCH}_{2}\right), 52.09,52.41\left(2 \times \mathrm{CO}_{2} \mathrm{CH}_{3}\right), 67.63,69.21,72.09,72.21,72.31$, $72.65,73.50,73.52,74.94,77.24,78.32,78.35$, [(4 $\left.\left.\times \mathrm{CH}_{2} \mathrm{C}_{6} \mathrm{H}_{5}\right),\left(\mathrm{C} 2^{\prime}-\mathrm{C} 5 '\right),(\mathrm{C} 1-\mathrm{C} 5)\right], 102.02$ (C- 
$\left.1^{\prime}\right), 116.95\left(-\mathrm{CH}_{2} \mathrm{CH}=\mathrm{CH}_{2}\right), 134.63\left(-\mathrm{CH}_{2} \mathrm{CHCH}_{2}\right), 127.09-138.20\left(\mathrm{CH}_{2} \mathrm{C}_{6} \mathrm{H}_{5}\right), 168.98,169.95$, $171.00(3 \times \mathrm{C}=\mathrm{O}) ; \mathrm{FAB}[\mathrm{M}+\mathrm{Na}]^{+}: m / z 847$.

Glycosylation reactions of D-galacturonate acceptor $(5)$ with D-galacuronates donors $(14 \alpha / \beta)$ A mixture of glycosyl acceptor 5 (195 mg, $0.4 \mathrm{mmol}$ ), glycosyl donors 14 $\alpha / \beta$ (270 mg, 0.5

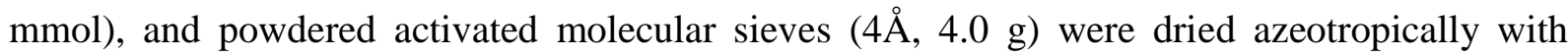
toluene, and then subjected to high vacuum for $2 \mathrm{~h}$. The mixture was dissolved in dry dichloromethane $(8 \mathrm{ml})$, and stirred for $2 \mathrm{~h}$ under argon at room temperature. After cooling to -70 ${ }^{\circ} \mathrm{C}$, TMSOTf $(83 \mu \mathrm{L}, 0.5 \mathrm{mmol})$ was added, and stirring was continued for $3 \mathrm{~h}$ at that temperature. The reaction mixture was then allowed to warm-up to room temperature and stirring was continued for an additional $18 \mathrm{~h}$ (TLC eluent $\mathrm{F}, \mathrm{R}_{f}$. 0.68 ). The reaction mixture was passed through a layer of alkaline alumina by elution with chloroform. The eluate was concentrated to $(30 \mathrm{~mL})$ then was washed with cold aq sat $\mathrm{NaHCO}_{3}(2 \times 15 \mathrm{~mL})$, and ice-water $(2 \times 15 \mathrm{~mL})$, dried and concentrated. The crude disaccharides were purified by HPLC (eluent ethyl acetate gradient $0 \% \rightarrow 25 \%$ in petrol ether v/v) to yield a mixture of $16 \alpha / \beta(175 \mathrm{mg}, 44 \%)$ in the ratio 3:1.

Benzyl (benzyl 4- $O$-acetyl-2,3-di- $O$-benzyl- $\alpha / \beta$-D-galactopyranosyluronate)-( $\rightarrow$ 4)-2,3-di$\boldsymbol{O}$-benzyl-1-deoxy-1-(prop-2-enyl)- $\alpha$-D-galactopyranuronate $(\mathbf{1 6} \alpha / \beta) .{ }^{1} \mathrm{H}$ NMR $(500 \mathrm{MHz}$, $\left.\mathrm{CDCl}_{3}\right) \delta 1.83,1.85\left[2 \times \mathrm{s}, 6 \mathrm{H}, \mathrm{OCOCH}_{3}(\alpha, \beta)\right], 2.29-2.35,2.42-2.49\left[2 \times \mathrm{m}, 4 \mathrm{H},-\mathrm{CH}_{2} \mathrm{CHCH}_{2}\right.$ $(\alpha, \beta)], 3.52-5.27(\mathrm{~m}, 54 \mathrm{H}), 4.74\left(\mathrm{~d}, 1 \mathrm{H}, J_{1^{\prime}, 2^{\prime}} 6.5 \mathrm{~Hz}, \mathrm{H}-1^{\prime} \beta\right), 5.01\left(\mathrm{~d}, 1 \mathrm{H}, J_{1^{\prime}, 2^{\prime}} 3.2 \mathrm{~Hz}, \mathrm{H}-1^{\prime} \alpha\right)$, 7.08-7.39 [m, 60H, - $\left.\mathrm{OCH}_{2} \mathrm{C}_{6} H_{5}(\alpha, \beta)\right]$; ${ }^{13} \mathrm{C} \mathrm{NMR}\left(125.7 \mathrm{MHz}, \mathrm{CDCl}_{3}\right) \delta 20.44,20.47[2 \times$ $\left.\mathrm{OCOCH}_{3}(\alpha, \beta)\right], 32.77,33.33\left[2 \times-\mathrm{CH}_{2} \mathrm{CHCH}_{2}(\alpha, \beta)\right], 60.27,66.46,66.79,67.23,67.38,71.77$, 72.12, 72.53, 72.81, 73.49, 73.59, $75.05\left[12 \times-\mathrm{OCH}_{2} \mathrm{C}_{6} \mathrm{H}_{5}(\alpha, \beta)\right], 67.45,68.56,69.77,71.37$, $72.33,72.65,74.34,74.43,75.20,75.55,75.84,77.18,78.37,78.49$ [(C-1)-(C-5) $\left.(\alpha, \beta),(\mathrm{C}-2)^{\prime}\right)$ $\left(\mathrm{C}-5^{\prime}\right)(\alpha, \beta), 4$ signals are isochronous], $99.02\left(\mathrm{C}-1^{\prime} \alpha\right), 102.31\left(\mathrm{C}-1^{\prime} \beta\right), 116.80,116.95[2 \times-$ $\left.\mathrm{CH}_{2} \mathrm{CHCH}_{2}(\alpha, \beta)\right], 126.86,127.06,127.36,127.41,127.63,127.67,127.73,127.83,127.88$, $128.09,128.25,128.39,128.48,128.72,128.84,129.21,134.53,134.65,134.71,134.97,135.25$, $135.90,137.52,137.60,137.91,137.93,138.07,138.29,138.55\left[-\mathrm{CH}_{2} \mathrm{CHCH}_{2}(\alpha, \beta),-\mathrm{OCH}_{2} \mathrm{C}_{6} \mathrm{H}_{5}\right.$ $(\alpha, \beta), 23$ signals are isochronous], 166.11, 166.80, 169.59, 169.79, 169.82, $171.01[6 \times \mathrm{C}=\mathrm{O}$ $(\alpha, \beta)]$; FAB $[\mathrm{M}+\mathrm{Li}]^{+}: m / z$ 983. Anal.calcd for $\mathrm{C}_{59} \mathrm{H}_{60} \mathrm{O}_{13}$ (977.10): C, 72.52; H, 6.19. Found: $\mathrm{C}$, $72.25 ; \mathrm{H}, 6.15$.

2,3-Di- $\boldsymbol{O}$-acetyl-1,2- $\boldsymbol{O}$-(1-ethoxyethylidene)- $\boldsymbol{\beta}$-L-rhamnopyranose (18). To a mixture of 2,3,4-Tri- $O$-acetyl- $\alpha$-L-rhamnopyranosyl bromide ${ }^{14} 17$ (6.0 g, $\left.18.9 \mathrm{mmol}\right), 2,4,6$-collidine (2.9 $\mathrm{mL}, 163.7 \mathrm{mmol})$, and tetrabutylammonium bromide $(2.4 \mathrm{~g}, 2.4 \mathrm{mmol})$ in $\mathrm{CH}_{2} \mathrm{Cl}_{2}(45 \mathrm{~mL})$, EtOH (anhydrous, $2.1 \mathrm{~mL}, 24 \mathrm{mmol}$ ) was added. The reaction mixture was stirred under argon for $9 \mathrm{~h}$ at room temperature (TLC eluent $\mathrm{L}, \mathrm{R}_{f}$. 0.64). Chloroform (100 mL) was added; the organic solution was washed with ice-water, dried and concentrated. The excess of collidine was removed under vacuum, and the resulting syrup was purified by flash chromatography (eluent ethyl acetate gradient $0 \% \rightarrow 25 \%$ in petrol ether - pyridine $0.05 \% \mathrm{v} / \mathrm{v} / \mathrm{v})$ to give $18(4.4 \mathrm{~g}, 81 \%)$ : mp 86-88 ${ }^{\circ} \mathrm{C} ;[\alpha]_{\mathrm{D}}{ }^{22}+32.7$ ( c 1.0, chloroform). Lit. $^{82}$; mp 87-88 ${ }^{\circ} \mathrm{C} ;[\alpha]_{\mathrm{D}}{ }^{22}+35.1(c 0.82$, chloroform). 
2,3-Di- $\boldsymbol{O}$-benzyl-1,2- $\boldsymbol{O}$-(1-ethoxyethylidene)- $\boldsymbol{\beta}$-L-rhamnopyranose (20). Compound 19 (4.40 $\mathrm{g}, 13.8 \mathrm{mmol})$ was dissolved in dry toluene $(20 \mathrm{~mL})$ and potassium hydroxide $(9.60 \mathrm{~g}$, powder) was added. The mixture was heated to reflux, and benzyl chloride $(14.5 \mathrm{~mL})$ was added dropwise. The reaction mixture was heated under reflux for $2.5 \mathrm{~h}$ and cooled to room temperature, and ice-water was added. The organic phase was diluted with toluene and washed with ice-water until neutral. The toluene solution was concentrated to an oil, which was then shifted to flash chromatography (eluent $\mathrm{G}, \mathrm{R}_{f} 0.61$ ) to give a syrupy product, which crystallized from petrol ether-ethyl acetate to give $20(4.7 \mathrm{~g}, 82 \%)$ as colorless crystals: mp $76-78{ }^{\circ} \mathrm{C} ;[\alpha]_{\mathrm{D}}{ }^{22}$ +2.9 (c 1.0 chloroform); ${ }^{1} \mathrm{H} \quad \mathrm{NMR}\left(500 \mathrm{MHz}, \mathrm{CDCl}_{3}\right) \delta 1.21[\mathrm{t}, 3 \mathrm{H}, \quad J \quad 6.9 \mathrm{~Hz}$, $\left.\mathrm{C}\left(\mathrm{OCH}_{2} \mathrm{CH}_{3}\right) \mathrm{CH}_{3}\right], 1.31\left(\mathrm{~d}, 3 \mathrm{H}, J 6.3 \mathrm{~Hz}, \mathrm{CH}_{3}-5\right), 1.73$ [s, 3H, C( $\left.\left.\mathrm{OCH}_{2} \mathrm{CH}_{3}\right) \mathrm{CH}_{3}\right], 3.31(\mathrm{~m}, 1 \mathrm{H}$, $\mathrm{H}-5), 3.48$ (t, $\left.1 \mathrm{H}, J_{4,5} 18.29 \mathrm{~Hz}, \mathrm{H}-4\right), 3.55$ [m, 2H, C( $\left.\left.\mathrm{OCH}_{2} \mathrm{CH}_{3}\right) \mathrm{CH}_{3}\right], 3.67$ (dd, 1H, J J,4 9.15 $\mathrm{Hz}, \mathrm{H}-3), 4.36\left(\mathrm{dd}, 1 \mathrm{H}, J_{2,3}=4.1 \mathrm{~Hz}, \mathrm{H}-2\right), 4.67$ (d, $\left.1 \mathrm{H}, J 11.03 \mathrm{~Hz}, \mathrm{CH}_{2} \mathrm{C}_{6} \mathrm{H}_{5}\right), 4.76(\mathrm{~s}, 2 \mathrm{H}$, $\left.\mathrm{CH}_{2} \mathrm{C}_{6} \mathrm{H}_{5}\right), 4.95$ (d, $\left.1 \mathrm{H}, J 11.04 \mathrm{~Hz}, \mathrm{CH}_{2} \mathrm{C}_{6} \mathrm{H}_{5}\right), 5.26$ (d, 1H, J $\left.J_{1,2} 2.52 \mathrm{~Hz}, \mathrm{H}-1\right), 7.29$ - 7.39 (m, $\left.10 \mathrm{H}, \mathrm{C}_{6} \mathrm{H}_{5}\right) ;{ }^{13} \mathrm{C} \mathrm{NMR}\left(125.7 \mathrm{MHz}, \mathrm{CDCl}_{3}\right) \delta 15.30\left[\mathrm{C}\left(\mathrm{OCH}_{2} \mathrm{CH}_{3}\right) \mathrm{CH}_{3}\right], 18.00\left(\mathrm{CH}_{3}-5\right), 24.70$

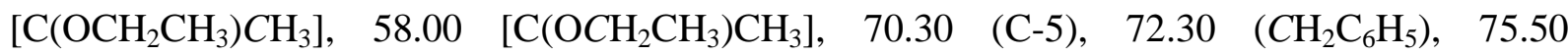
$\left(\mathrm{CH}_{2} \mathrm{C}_{6} \mathrm{H}_{5}\right), 77.03(\mathrm{C}-2), 79.10(\mathrm{C}-3), 79.60(\mathrm{C}-4), 97.30(\mathrm{C}-1), 123.60$ [C(OEt)CH$\left.)_{3}\right], 127.80$, $127.90,128.00,128.10,128.40,128.50,137.90,138.30\left(\mathrm{C}_{6} \mathrm{H}_{5}\right.$ four signals are isochronous $)$. Anal.Calcd for $\mathrm{C}_{24} \mathrm{H}_{30} \mathrm{O}_{6}$ (414.49): C, 69.54; H, 7.30. Found: C, 69.17; H, 7.33.

Crystal data. Mol. formula $\mathrm{C}_{24} \mathrm{H}_{30} \mathrm{O}_{6}$; Formula weight: 414.48; temperature: $173(2) \mathrm{K}$; wavelength: $0.71073 \AA$; crystal system: Orthorhombic; space group (H.-M.): $\mathrm{P} 2{ }_{1} 2_{1} 2_{1}$; space group (Hall): P 2ac 2ab; Unit cell dimensions: $\mathrm{a}=10.4095(9) \AA, \alpha=90^{\circ}, \mathrm{b}=11.4518(11) \AA, \beta=$ $90^{\circ}, \mathrm{c}=18.9505(13) \AA, \gamma=90^{\circ}$; volume: $2259.0(3) \AA^{3} ; \mathrm{Z}=4$; density (calculated): 1.219 $\mathrm{Mg} / \mathrm{m}^{3}$; absorption coefficient : $0.087 \mathrm{~mm}^{-1} ; \mathrm{F}(000): 888$; crystal size: $0.60 \times 0.40 \times 0.15 \mathrm{~mm}^{3}$; $\Theta$ range for data collection: 2.91 to $22.49^{\circ}$; index ranges: $-11 \leq \mathrm{h} \leq 11,-12 \leq \mathrm{k} \leq 12,-18 \leq 1 \leq 20$; reflections collected: 13542; independent reflections: 2935 [R(int $)=0.0303$ ]; completeness to $\Theta$ $=22.49^{\circ}: 98.8 \%$; absorption correction: none; max. and min. transmission: 0.9871 and 0.9498; refinement method: full-matrix least-squares on F2; data / restraints / parameters: 2935 / 6 / 312; goodness-of-fit on F2: 1.113; final R indices: $[\mathrm{I}>2 \sigma(\mathrm{I})]$; R1 $=0.0622$, wR2 $=0.1695$; $\mathrm{R}$ indices (all data): $\mathrm{R} 1=0.0658, \mathrm{wR} 2=0.1717$; absolute structure parameter: $0(2)$; extinction coefficient : $0.020(3)$; largest diff. peak and hole: 0.183 and -0.195 e. $\AA^{-3}$.

Hydrolysis of compound 20. 2,3-Di- $O$-benzyl-1,2- $O$-(1-ethoxyethylidene)- $\beta$-L-rhamnopyranose. Compound 20 (4.4 g, $10.61 \mathrm{mmol})$ was hydrolysed by sequential treatment with $70 \%$ aqueous acetic acid $\left(28 \mathrm{~mL}\right.$ ) at room temperature for $7 \mathrm{~min}$ (TLC eluent $\mathrm{K}, \mathrm{R}_{f} 0.52$ ). The solution was then concentrated and acetic acid in the syrupy residue was removed by co-evaporation with toluene. The ${ }^{1} \mathrm{H}$ NMR spectrum indicated the product to be essentially pure. Without further purification, the crude syrupy residue was dissolved in dry pyridine $(106.5 \mathrm{~mL})$. Acetic anhydride $(53 \mathrm{~mL})$ was added at $0{ }^{\circ} \mathrm{C}$ and the reaction mixture stirred at room temperature for $1 \mathrm{~h}$ (TLC eluent $\mathrm{I}, \mathrm{R}_{f}$ 0.71). The mixture was poured into ice-water and the aqueous layer extracted with chloroform. The combined organic extracts was washed with cold $1 \% \mathrm{HCl}$, ice-water, cold 
sat aq $\mathrm{NaHCO}_{3}$, then again with ice-water, dried and concentrated. The syrupy residue was purified by flash chromatography (eluent $\mathrm{A}$ ).

1,2-Di- $\boldsymbol{O}$-acetyl-3,4-di- $\boldsymbol{O}$-benzyl- $\boldsymbol{\alpha}$-L-rhamnopyranose $(\mathbf{2 1} \alpha)$. Colourless amorphous solid (3.01 g, 66\%): $[\alpha]_{\mathrm{D}}{ }^{21}-20.8$ (c 1.0, chloroform); ${ }^{1} \mathrm{H} \mathrm{NMR}\left(500 \mathrm{MHz}, \mathrm{CDCl}_{3}\right) \delta 1.34(\mathrm{~d}, 3 \mathrm{H}, J$ $\left.6.31 \mathrm{~Hz}, \mathrm{CH}_{3}-5\right), 2.08,2.17$ (2s, 6H, $\left.\mathrm{OCOCH}_{3}\right), 3.49$ (t, 1H, $\left.J_{4,5} 9.5 \mathrm{~Hz}, \mathrm{H}-4\right), 3.81$ (m, 1H, H-5), $3.94\left(\mathrm{dd}, 1 \mathrm{H}, J_{3,4} 9.46 \mathrm{~Hz}, \mathrm{H}-3\right), 4.55\left(\mathrm{~d}, 1 \mathrm{H}, J 11.04, \mathrm{CH}_{2} \mathrm{C}_{6} \mathrm{H}_{5}\right), 4.65(\mathrm{~d}, 1 \mathrm{H}, J 10.92$, $\left.\mathrm{CH}_{2} \mathrm{C}_{6} \mathrm{H}_{5}\right), 4.75\left(\mathrm{~d}, 1 \mathrm{H}, J 11.04, \mathrm{CH}_{2} \mathrm{C}_{6} \mathrm{H}_{5}\right), 4.93\left(\mathrm{~d}, 1 \mathrm{H}, J 10.73, \mathrm{CH}_{2} \mathrm{C}_{6} \mathrm{H}_{5}\right), 5.36\left(\mathrm{dd}, 1 \mathrm{H}, J_{2,3}\right.$ 2.84, H-2), 6.01 (d, $\left.1 \mathrm{H}, J_{1,2} 1.89 \mathrm{~Hz}, \mathrm{H}-1\right), 7.29-7.37\left(\mathrm{~m}, 10 \mathrm{H}, \mathrm{CH}_{2} \mathrm{C}_{6} H_{5}\right) ;{ }^{13} \mathrm{C}$ NMR $(125.7$ $\left.\mathrm{MHz}, \mathrm{CDCl}_{3}\right) \delta 17.95\left(\mathrm{CH}_{3}-5\right), 20.81,20.87\left(2 \times \mathrm{COCH}_{3}\right), 67.80(\mathrm{C}-2), 70.00(\mathrm{C}-5), 71.90$, $75.50\left(2 \times \mathrm{CH}_{2} \mathrm{C}_{6} \mathrm{H}_{5}\right), 77.60(\mathrm{C}-3), 79.50(\mathrm{C}-4), 91.11(\mathrm{C}-1), 127.76,127.89,127.90,128.00$, 128.40, 137.70, $138.20\left(\mathrm{CH}_{2} \mathrm{C}_{6} \mathrm{H}_{5}\right.$ seven signals are isochronous $), 168.40,169.90\left(2 \times \mathrm{COCH}_{3}\right)$. Anal. calcd. for $\mathrm{C}_{24} \mathrm{H}_{28} \mathrm{O}_{7}$ (428.47): C, 67.28; H, 6.59. Found: C, 67.29; H, 6.59.

1,2-Di- $\boldsymbol{O}$-acetyl-3,4-di- $\boldsymbol{O}$-benzyl- $\boldsymbol{\beta}$-L-rhamnopyranose $(\mathbf{2 1} \boldsymbol{\beta})$. Colorless syrup $(0.62 \mathrm{~g}, 14 \%)$ : $[\alpha]_{\mathrm{D}}{ }^{21}+29.2\left(c\right.$ 1, chloroform); ${ }^{1} \mathrm{H} \mathrm{NMR}\left(500 \mathrm{MHz} \mathrm{CDCl}_{3}\right) \delta 1.38\left(\mathrm{~d}, 3 \mathrm{H}, J=5.99 \mathrm{~Hz}, \mathrm{CH}_{3}-5\right)$, 2.09, $2.22\left(2 \mathrm{~s}, 6 \mathrm{H}, \mathrm{OCOCH}_{3}\right), 3.44$ (t, $\left.1 \mathrm{H}, J_{4,5} 9.5 \mathrm{~Hz}, \mathrm{H}-4\right), 3.48$ - 3.55 (m, 1H, H-5), 3.70 (dd, $\left.1 \mathrm{H}, J_{3,4} 9.15 \mathrm{~Hz}, \mathrm{H}-3\right), 4.50\left(\mathrm{~d}, 1 \mathrm{H}, J 11.04, \mathrm{CH}_{2} \mathrm{C}_{6} \mathrm{H}_{5}\right), 4.62\left(\mathrm{~d}, 1 \mathrm{H}, J 11.03, \mathrm{CH}_{2} \mathrm{C}_{6} \mathrm{H}_{5}\right), 4.72(\mathrm{~d}$, $1 \mathrm{H}, J$ 11.03, $\mathrm{CH}_{2} \mathrm{C}_{6} \mathrm{H}_{5}$ ), $4.92\left(\mathrm{~d}, 1 \mathrm{H}, J 11.04, \mathrm{CH}_{2} \mathrm{C}_{6} \mathrm{H}_{5}\right.$ ), 5.61 (dd, 1H, J2,3 3.47, H-2), 5.73 (d, $\left.1 \mathrm{H}, J_{1,2} 1.27 \mathrm{~Hz}, \mathrm{H}-1\right), 7.27-7.37\left(\mathrm{~m}, 10 \mathrm{H}, \mathrm{C}_{6} H_{5}\right) ;{ }^{13} \mathrm{C} \mathrm{NMR}\left(125.7 \mathrm{MHz}, \mathrm{CDCl}_{3}\right)$ $\delta 17.80\left(\mathrm{CH}_{3}-5\right), 20.70,20.90\left(2 \times \mathrm{COCH}_{3}\right), 67.50(\mathrm{C}-2), 71.50,75.40\left(2 \times \mathrm{CH}_{2} \mathrm{C}_{6} \mathrm{H}_{5}\right), 72.70$ (C-5), 79.20 (C-3), 79.60 (C-4), 91.10 (C-1), 127.70, 127.80, 127.90, 128.00, 128.30, 128.40, $137.40,138.10\left(\mathrm{CH}_{2} \mathrm{C}_{6} \mathrm{H}_{5}\right.$ six signals are isochronous $), 168.60,170.50\left(2 \times \mathrm{COCH}_{3}\right)$.

2- $\boldsymbol{O}$-Acetyl-1-bromo-3,4-di- $\boldsymbol{O}$-benzyl- $\boldsymbol{\alpha}$-L-rhamnopyranose (22). To a solution of $21 \alpha / \beta$ (500 $\mathrm{mg}, 1.17 \mathrm{mmol})$ in dry dichloromethane $(7 \mathrm{~mL})$, was added oxalyl bromide ${ }^{70,78}(162 \mu \mathrm{l})$ was added under argon at $-40{ }^{\circ} \mathrm{C}$. After an additional one hour at that temperature, the reaction mixture was kept at room temperature for one hour (TLC eluent $\mathrm{F}, \mathrm{R}_{f} 0.75$ ). The mixture was concentrated and repeatedly co-evaporated with toluene to give crude product of $20(500 \mathrm{mg}, 96$ $\%)$. The compound 20 was unstable for storage and was used directly without further purification.

Benzyl (2-O-acetyl-3,4-di- $O$-benzyl- $\alpha$-L-rhamnopyranosyl)-(1 $\rightarrow 4)$-2,3-di- $O$-benzyl-1-deoxy1-(prop-2-enyl)- $\boldsymbol{\alpha}$-D-galactopyranuronate (23). (a) Via 21. Glycosyl acceptor 5 (0.4 mmol), glycosyl donor $19(\alpha / \beta)(0.5 \mathrm{mmol})$, and powdered activated molecular sieves $(4 \AA$, $4.0 \mathrm{~g})$ were dried azeotropically with toluene, and then subjected to high vacuum for $2 \mathrm{~h}$. The mixture was dissolved in dry dichloromethane $(8 \mathrm{~mL})$, and the reaction mixture was stirred for $2 \mathrm{~h}$ under argon at room temperature in the dark. After cooling to $-70^{\circ} \mathrm{C}$, TMSOTf $(83 \mu \mathrm{l}, 0.5 \mathrm{mmol})$ was added, and stirring was continued for $3 \mathrm{~h}$ at that temperature. The reaction mixture was then allowed to warmup to room temperature and stirring was continued for an additional $18 \mathrm{~h}$ (TLC eluent F). The reaction mixture was passed through a layer of alkaline alumina by elution with chloroform. The eluate was concentrated to $30 \mathrm{~mL}$ and then washed with cold aq sat $\mathrm{NaHCO}_{3}(2$ $\times 15 \mathrm{~mL})$, ice-water $(2 \times 15 \mathrm{~mL})$, dried and concentrated. The crude residue was purified by HPLC (eluent ethyl acetate gradient $0 \% \rightarrow 25 \%$ in petrol ether v/v) to give disaccharide $23\left(\mathrm{R}_{f}\right.$ 
$0.55,18 \%)$, donor $21\left(\mathrm{R}_{f} 0.48,26 \%\right)$, and accompanying transesterification product of the acceptor $5\left(\mathrm{R}_{f} 0.68,23 \%\right)$. The NMR data of $\mathbf{2 5}$ supported the chemical structure shown in Scheme 4.

(b) Via 22. Glycosyl acceptor $5(0.4 \mathrm{mmol})$, glycosyl donor $22(0.5 \mathrm{mmol})$, powdered activated molecular sieves $(4 \AA, 4.0 \mathrm{~g})$, and 2,6-di-tert-butyl-4-methylpyridine (0.6 mmol) were dried under high vacuum at ambient temperature for $2 \mathrm{~h}$. The solids were then suspended in dry dichloromethane $(8 \mathrm{~mL})$, and the reaction mixture was stirred for $2 \mathrm{~h}$ under argon at room temperature in the dark. Subsequently, after chilling to $-70^{\circ} \mathrm{C}$, silver trifluoromethanesulfonate (141 $\mathrm{mg}, 0.55 \mathrm{mmol}$ ) was added. After stirring for $2 \mathrm{~h}$ at that temperature, the chilling was terminated and the mixture was stirred for $18 \mathrm{~h}$ at ambient temperature (TLC eluent $\mathrm{B}, \mathrm{R}_{f} 0.54$ ). The reaction mixture was then passed through a layer of alkaline alumina by elution with chloroform. The eluate was concentrated to $(30 \mathrm{~mL})$ and then washed with cold aq sat $\mathrm{NaHCO}_{3}$ $(2 \times 15 \mathrm{~mL})$, ice-water $(2 \times 15 \mathrm{~mL})$, dried and concentrated. The residue was purified by HPLC (eluent ethyl acetate gradient $0 \% \rightarrow 25 \%$ in petrol ether v/v) to give the desired disaccharide 23 in 54\% yield as a colourless syrup, ${ }^{1} \mathrm{H} \mathrm{NMR}\left(500 \mathrm{MHz}, \mathrm{CDCl}_{3}\right) \delta 1.28\left[\mathrm{~d}, 3 \mathrm{H}, J 6.4 \mathrm{~Hz}, \mathrm{CH}_{3}\right.$ (Rha)], 2.13 [s, 3H, $\mathrm{OCOCH}_{3}$ (Rha)], $2.41\left(\mathrm{~m}, 1 \mathrm{H}, \mathrm{CH}_{2} \mathrm{CHCH}_{2}\right), 3.40\left[\mathrm{t}, 1 \mathrm{H}, J_{2,3}=J_{3,4}=9.5 \mathrm{~Hz}\right.$, H-4 (Rha)], 3.64 [s, 3H, $\mathrm{CO}_{2} \mathrm{CH}_{3}$ (GalA)], 3.71 - 3.78 [m, 2H, H-3 (GalA), H-5 (Rha)], 3.83 [dd, $1 \mathrm{H}, J_{1,2} 4.5 \mathrm{~Hz}, J_{2,3} 7.3 \mathrm{~Hz}, \mathrm{H}-2$ (GalA)], 3.88 [dd, $1 \mathrm{H}, J_{2,3} 3.3 \mathrm{~Hz}, J_{3,4} 9.5 \mathrm{~Hz}, \mathrm{H}-3$ (Rha)], 4.28 [d, 1H, $J_{4,5} 3.5 \mathrm{~Hz}, \mathrm{H}-5$ (GalA)], 4.37 - 4.41 [m, 1H, H-1 (GalA)], 4.45 [dd, 1H, $J_{3,4} 2.5 \mathrm{~Hz}, \mathrm{H}-4$ $(\mathrm{GalA})], 4.47\left(\mathrm{~d}, 1 \mathrm{H}, J 11.6 \mathrm{~Hz}, \mathrm{OCH}_{2} \mathrm{C}_{6} \mathrm{H}_{5}\right), 4.57$ - $4.68\left(\mathrm{~m}, 5 \mathrm{H}, \mathrm{OCH}_{2} \mathrm{C}_{6} \mathrm{H}_{5}\right), 4.73(\mathrm{~d}, 1 \mathrm{H}, J$ $\left.12.0 \mathrm{~Hz}, \mathrm{OCH}_{2} \mathrm{C}_{6} \mathrm{H}_{5}\right), 4.91$ (d, $1 \mathrm{H}, J 11.3 \mathrm{~Hz}, \mathrm{OCH}_{2} \mathrm{C}_{6} \mathrm{H}_{5}$ ), 5.05 [d, $1 \mathrm{H}, J_{1,2} 1.5 \mathrm{~Hz}, \mathrm{H}-1$ (Rha)], 5.05 - $5.14\left(4 \mathrm{~m}, 2 \mathrm{H}, \mathrm{CH}_{2} \mathrm{CH}=\mathrm{CH}_{2}\right), 5.45$ [dd, $1 \mathrm{H}, J_{1,2} 1.8 \mathrm{~Hz}, J_{2,3} 3.3 \mathrm{~Hz}, \mathrm{H}-2$ (Rha)], 5.83 (m, $\left.1 \mathrm{H}, \mathrm{CH}_{2} \mathrm{CH}=\mathrm{CH}_{2}\right), 7.23-7.36\left(\mathrm{~m}, 20 \mathrm{H}, \mathrm{OCH}_{2} \mathrm{C}_{6} H_{5}\right) ;{ }^{13} \mathrm{C} \mathrm{NMR}\left(125.7 \mathrm{MHz}, \mathrm{CDCl}_{3}\right) \delta 17.95$ $\left[\mathrm{CH}_{3}\right.$ (Rha) $], 21.06\left[\mathrm{OCOCH}_{3}\right.$ (Rha)], $31.61\left(\mathrm{CH}_{2} \mathrm{CH}=\mathrm{CH}_{2}\right), 51.96\left(\mathrm{CO}_{2} \mathrm{CH}_{3}\right), 68.43[\mathrm{C}-5$ (Rha)], 69.02 [C-2 (Rha)], 71.67 [C-5 (GalA)], $71.70\left(\mathrm{OCH}_{2} \mathrm{C}_{6} \mathrm{H}_{5}\right), 72.89\left(\mathrm{OCH}_{2} \mathrm{C}_{6} \mathrm{H}_{5}\right), 72.97$ [C-1 (GalA)], $73.38\left(\mathrm{OCH}_{2} \mathrm{C}_{6} \mathrm{H}_{5}\right), 73.70$ [C-4 (GalA)], $74.93\left(\mathrm{OCH}_{2} \mathrm{C}_{6} \mathrm{H}_{5}\right), 76.01$ [C-2 (GalA)], 76.55 [C-3 (GalA)], 77.63 [C-3 (Rha)], 79.69 [C-4 (Rha)], 98.26 [C-1 (Rha], 116.93 $\left(\mathrm{CH}_{2} \mathrm{CH}=\mathrm{CH}_{2}\right), 127.42,127.54,127.59,127.66,127.82,127.91,128.00,128.05,128.08,128.19$, $128.29,128.38,128.43,137.95,138.05,138.08,138.76\left(\mathrm{OCH}_{2} \mathrm{C}_{6} \mathrm{H}_{5}\right.$ seven signals are isochronous), $134.62\left(\mathrm{CH}_{2} \mathrm{CH}=\mathrm{CH}_{2}\right), 169.07\left[\mathrm{CO}_{2} \mathrm{CH}_{3}(\mathrm{GalA})\right], 170.06\left[\mathrm{OCO}_{3} \mathrm{CH}_{3}(\mathrm{Rha})\right] ; \mathrm{m} / \mathrm{z}$ 879.37216 [M+Na] $]^{+}$. Calc. For $\mathrm{C}_{52} \mathrm{H}_{56} \mathrm{O}_{11} \mathrm{M}+\mathrm{Na}: \mathrm{m} / \mathrm{z}$, 879.37201. Anal.calcd for $\mathrm{C}_{52} \mathrm{H}_{56} \mathrm{O}_{11}$ (856.99): C, 72.88; H, 6.59. Found: C, 72.70; H, 6.54.

\section{Methyl (2-O-acetyl-3,4-di- $O$-benzyl- $\alpha$-L-rhamnopyranosyl)-(1 $\rightarrow$ 4)-2,3-di- $O$-benzyl-1-} deoxy-1-(prop-2-enyl)- $\alpha$-D-galactopyranuronate (24)

Glycosyl acceptor 4 (0.4 mmol), glycosyl donor $22(0.5 \mathrm{mmol})$, powdered activated molecular sieves (4 ̊., $4.0 \mathrm{~g})$, and 2,6-di-tert-butyl-4-methylpyridine $(0.6 \mathrm{mmol})$ were dried under high vacuum at ambient temperature for $2 \mathrm{~h}$. The solids were then suspended in dry dichloromethane $(8 \mathrm{~mL})$, and the reaction mixture was stirred for $2 \mathrm{~h}$ under argon at room temperature in the dark. Subsequently, after chilling to $-70^{\circ} \mathrm{C}$, silver trifluoromethanesulfonate $(141 \mathrm{mg}, 0.55 \mathrm{mmol})$ was added. After stirring for $2 \mathrm{~h}$ at that temperature, the chilling was terminated and the mixture was 
stirred for $18 \mathrm{~h}$ at ambient temperature (TLC eluent $\mathrm{F}, \mathrm{R}_{f} 0.77$ ). The reaction mixture was then passed through a layer of alkaline alumina by elution with chloroform. The eluate was concentrated to $(30 \mathrm{~mL})$ and then washed with cold aq sat $\mathrm{NaHCO}_{3}(2 \times 15 \mathrm{~mL})$, ice-water $(2 \times$ $15 \mathrm{~mL}$ ), dried and concentrated. After HPLC chromatography (eluent ethyl acetate gradient $0 \%$ $\rightarrow 25 \%$ in petrol ether v/v) the desired disaccharide $\mathbf{2 4}$ was obtained in $57 \%$ yield as a colorless syrup; $[\alpha]_{\mathrm{D}}{ }^{21}+29.6$ (c 1.0, in chloroform); ${ }^{1} \mathrm{H} \mathrm{NMR}\left(500 \mathrm{MHz}, \mathrm{CDCl}_{3}\right) \delta 1.28[\mathrm{~d}, 3 \mathrm{H}, J 6.4 \mathrm{~Hz}$, $\mathrm{CH}_{3}$ (Rha)], 2.13 [s, 3H, $\mathrm{OCOCH}_{3}$ (Rha)], $2.41\left(\mathrm{~m}, 1 \mathrm{H}, \mathrm{CH}_{2} \mathrm{CH}=\mathrm{CH}_{2}\right), 3.40\left[\mathrm{t}, 1 \mathrm{H}, J_{2,3}=J_{3,4}=\right.$ $9.5 \mathrm{~Hz}, \mathrm{H}-4$ (Rha)], 3.64 [s, 3H, $\mathrm{CO}_{2} \mathrm{CH}_{3}$ (GalA)], 3.71 - 3.78 [m, 2H, H-3 (GalA), H-5 (Rha)], $3.83\left[\mathrm{dd}, 1 \mathrm{H}, J_{1,2} 4.5 \mathrm{~Hz}, J_{2,3} 7.3 \mathrm{~Hz}, \mathrm{H}-2\right.$ (GalA)], 3.88 [dd, 1H, $J_{2,3} 3.3 \mathrm{~Hz}, J_{3,4} 9.5 \mathrm{~Hz}, \mathrm{H}-3$ (Rha)], 4.28 [d, 1H, $J_{4,5} 3.5 \mathrm{~Hz}, \mathrm{H}-5$ (GalA)], 4.37-4.41 [m, 1H, H-1 (GalA)], 4.45 [dd, 1H, $J_{3,4}$ $2.5 \mathrm{~Hz}, \mathrm{H}-4(\mathrm{GalA})], 4.47\left(\mathrm{~d}, 1 \mathrm{H}, J 11.6 \mathrm{~Hz}, \mathrm{OCH}_{2} \mathrm{C}_{6} \mathrm{H}_{5}\right), 4.57-4.68\left(\mathrm{~m}, 5 \mathrm{H}, \mathrm{OCH}_{2} \mathrm{C}_{6} \mathrm{H}_{5}\right), 4.73$ $\left(\mathrm{d}, 1 \mathrm{H}, J 12.0 \mathrm{~Hz}, \mathrm{OCH}_{2} \mathrm{C}_{6} \mathrm{H}_{5}\right), 4.91\left(\mathrm{~d}, 1 \mathrm{H}, J 11.3 \mathrm{~Hz}, \mathrm{OCH}_{2} \mathrm{C}_{6} \mathrm{H}_{5}\right), 5.05$ [d, $1 \mathrm{H}, J_{1,2} 1.5 \mathrm{~Hz}, \mathrm{H}-1$ (Rha)], 5.05 - $5.14\left(4 \mathrm{~m}, 2 \mathrm{H}, \mathrm{CH}_{2} \mathrm{CH}=\mathrm{CH}_{2}\right), 5.45$ [dd, $1 \mathrm{H}, J_{1,2} 1.8 \mathrm{~Hz}, J_{2,3} 3.3 \mathrm{~Hz}, \mathrm{H}-2$ (Rha)], $5.83\left(\mathrm{~m}, 1 \mathrm{H}, \mathrm{CH}_{2} \mathrm{CH}=\mathrm{CH}_{2}\right), 7.23-7.36\left(\mathrm{~m}, 2 \mathrm{H}, \mathrm{OCH}_{2} \mathrm{C}_{6} \mathrm{H}_{5}\right) ;{ }^{13} \mathrm{C} \mathrm{NMR}\left(125.7 \mathrm{MHz}, \mathrm{CDCl}_{3}\right)$ $\delta 17.95\left[\mathrm{CH}_{3}(\mathrm{Rha})\right], 21.06\left[\mathrm{OCOCH}_{3}(\mathrm{Rha})\right], 31.61\left(\mathrm{CH}_{2} \mathrm{CH}=\mathrm{CH}_{2}\right), 51.96\left(\mathrm{CO}_{2} \mathrm{CH}_{3}\right), 68.43[\mathrm{C}-$ 5 (Rha)], 69.02 [C-2 (Rha)], 71.67 [C-5 (GalA)], $71.70\left(\mathrm{OCH}_{2} \mathrm{C}_{6} \mathrm{H}_{5}\right), 72.89\left(\mathrm{OCH}_{2} \mathrm{C}_{6} \mathrm{H}_{5}\right), 72.97$ [C-1 ( GalA)], $73.38\left(\mathrm{OCH}_{2} \mathrm{C}_{6} \mathrm{H}_{5}\right), 73.70$ [C-4 (GalA)], $74.93\left(\mathrm{OCH}_{2} \mathrm{C}_{6} \mathrm{H}_{5}\right), 76.01$ [C-2 (GalA)], 76.55 [C-3 (GalA)], 77.63 [C-3 (Rha)], 79.69 [C-4 (Rha)], 98.26 [C-1 (Rha], 116.93 $\left(\mathrm{CH}_{2} \mathrm{CH}=\mathrm{CH}_{2}\right), 127.42,127.54,127.59,127.66,127.82,127.91,128.00,128.05,128.08,128.19$, $128.29,128.38,128.43,137.95,138.05,138.08,138.76\left(\mathrm{OCH}_{2} \mathrm{C}_{6} \mathrm{H}_{5}\right.$ seven signals are isochronous), $134.62\left(\mathrm{CH}_{2} \mathrm{CH}=\mathrm{CH}_{2}\right), 169.07\left[\mathrm{CO}_{2} \mathrm{CH}_{3}(\mathrm{GalA})\right], 170.06\left[\mathrm{OCOCH}_{3}(\mathrm{Rha})\right] ; \mathrm{m} / \mathrm{z}$ $803.34023[\mathrm{M}+\mathrm{Na}]^{+}$. Calc. for $\mathrm{C}_{46} \mathrm{H}_{52} \mathrm{O}_{11} \mathrm{Na}: m / z, 803.34076$. Anal.calcd for $\mathrm{C}_{46} \mathrm{H}_{52} \mathrm{O}_{11}$ (780.90): C, 70.75; H, 6.71. Found: C, 70.50; H, 6.54.

\section{Acknowledgements}

This work was supported by a grant of the Deutsche Forschungsgemeinschaft (DFG) and the University of Rostock.

\section{References}

1. Farouk A.; Vogel C., 22nd International Carbohydrate Symposium, Glasgow, U. K., 23 - 27 July, 2004. Abstract No. P380

2. Vogel, C.; Farouk, M.; Michalik, M.; Reinke, H.; Jarosz, S. Polish J. Chem. 2005, 79, 252265.

3. Vogel, C.; Nolting, B.; Kramer, S.; Steffan, W.; Ott, A.-J, Synthesis of Pectin Fragments by Modular Design Principle. In: Advances in Pectin and Pectinase Research, Vorhagen, F.; 
Schols, H.; Visser, R., Eds., Kluwer Academic Publishers: Dordrecht-Boston-London, 2003; p 209.

4. Schmidt, R. R.; Michel; J. Angew. Chem. Int. Ed. Engl. 1980, 19, 731-732. http://dx.doi.org/10.1002/anie.198007311

5. Nolting, B.; Boye, H.; Vogel, C. J. Carbohydr. Chem. 2001, 20, 585-610. http://dx.doi.org/10.1081/CAR-100108276

6. Magaud, D.; Grandjean, C.; Doutheau, A.; Anker, D.; Shevchik, V.; Cotte-Pattat, N.; RobertBaudouy; J. Tetrahedron Lett. 1997, 38, 241-244. http://dx.doi.org/10.1016/S0040-4039(96)02308-8

7. Magaud, D.; Grandjean, C.; Doutheau, A.; Anker, D.; Shevchik, V.; Cotte-Pattat, N.; RobertBaudouy, J. Carbohydr. Res. 1998, 314, 189-199.

http://dx.doi.org/10.1016/S0008-6215(98)00312-7

8. Ogawa, T.; Nakabayashi, S. Carbohydr. Res. 1981, 93, C1-C5

http://dx.doi.org/10.1016/S0008-6215(00)80764-8

9. Kramer, S.; Nolting, B.; Ott, A. J.; Vogel, C. J. Carbohydr. Chem. 2000, 19, 891-921. http://dx.doi.org/10.1080/07328300008544125

10. Crich, D. J. Carbohydr. Chem. 2002, 21, 667-690. http://dx.doi.org/10.1081/CAR-120016486

11. Pozsgay, V.; Pannell, L. Carbohydr. Res. 1994, 258, 105-122. http://dx.doi.org/10.1016/0008-6215(94)84079-2

12. Nolting, B.; Boye, H.; Vogel, C. J. Carbohyd. Chem. 2000, 19, 7, 89.

13. Toepfer, A.; Schmidt, R. R. J. Carbohydr. Chem. 1993, 12, 809-822. http://dx.doi.org/10.1080/07328309308020096

14. Betaneli, V. I.; Ovchinnikov, M. V.; Backinowsky, L. V.; Kochetkov, N. K. Carbohydr. Res. 1980, 84, 211-224. http://dx.doi.org/10.1016/S0008-6215(00)85552-4

15. Wang, W.; Kong, F. J. Carbohydr. Chem. 1999, 18, 263-273.; Lemieux, R. U.; Morgan, A. R. Can. J. Chem. 1965, 43, 2199-2204. 
16. Du, Y.; Kong, F. J. Carbohydr. Chem. 1999, 18, 655-666. http://dx.doi.org/10.1080/07328309908544027

17. Bognár, R.; Farkas, I. Liebigs Ann. Chem. 1990, 499-500. http://dx.doi.org/10.1002/jlac.199019900194

18. Vernay, H. F.; Rachaman, E. S.; Eby, R.; Schuerch, C. Carbohyd. Res. 1980, 78, 267-273. http://dx.doi.org/10.1016/0008-6215(80)90007-5

19. Perlin, A. S. Can. J. Chem. 1963, 41, 399-406. http://dx.doi.org/10.1139/v63-058

20. Perlin, A. S. Can. J. Chem. 1963, 41, 555-561. http://dx.doi.org/10.1139/v63-082

21. Soliman, S. E.; Bassily, R. W.; El-Sokkary, R. I.; Nashed, M. A. Carbohydr. Res. 2003, 338, 2337-2340. http://dx.doi.org/10.1016/S0008-6215(03)00317-3

22. Jacquinet, J.-C.; Sinaÿ, P. J. Chem. Soc., Perkin Trans. 1 1979, 314-318.

23. Paulsen, H.; Khan, S. H.; O'Neill, R. A., Eds.; Modern Methods in Carbohydrate Synthesis; Harwood Academic Publishers, 1996.

24. Vogel, C.; Kramer, S.; Ott, A. J. Liebigs Ann. Chem. 1997, 1425-1428.

25. Sinaÿ, P. Pure Appl. Chem. 1978, 50, 1437-1452.

http://dx.doi.org/10.1351/pac197850111437

26. Perrin, D. D.; Armarego, W. L. F.; Purification of Laboratory Chemicals 3rd Edition, Pergamon Press: Oxford, 1988. ISBN 0-08-034714-2. 